\title{
HYPERCYCLIC SUBSPACES ON FRÉCHET SPACES WITHOUT CONTINUOUS NORM
}

\author{
QUENTIN MENET
}

\begin{abstract}
Known results about hypercyclic subspaces concern either Fréchet spaces with a continuous norm or the space $\omega$. We fill the gap between these spaces by investigating Fréchet spaces without continuous norm. To this end, we divide hypercyclic subspaces into two types: the hypercyclic subspaces $M$ for which there exists a continuous seminorm $p$ such that $M \cap \operatorname{ker} p=\{0\}$ and the others. For each of these types of hypercyclic subspaces, we establish some criteria. This investigation permits us to generalize several results about hypercyclic subspaces on Fréchet spaces with a continuous norm and about hypercyclic subspaces on $\omega$. In particular, we show that each infinite-dimensional separable Fréchet space supports a mixing operator with a hypercyclic subspace.
\end{abstract}

\section{INTRODUCTION}

We denote by $\mathbb{Z}$ the set of integers, by $\mathbb{N}$ the set of positive integers and by $\mathbb{Z}_{+}$ the set of non-negative integers.

Let $\left(T_{k}\right)$ be a sequence of linear continuous operators from $X$ to $Y$ where $X$ is an infinite-dimensional Fréchet space and $Y$ is a separable topological vector space. The sequence $\left(T_{k}\right)$ is said to be hypercyclic if there exists a vector $x$ in $X$ (also called hypercyclic) such that the orbit of $x$ for $\left(T_{k}\right)$ is dense in $Y$. We are interested in the existence of closed infinite-dimensional subspaces in which every non-zero vector is hypercyclic. Such a subspace is called a hypercyclic subspace.

Some classical hypercyclic operators, like the translation operators on the space of entire functions, possess a hypercyclic subspace [7] but some others, like scalar multiples of the backward shift on $l^{p}$, do not possess any hypercyclic subspace [26]. A natural question is thus: "Which hypercyclic operators possess a hypercyclic subspace?". Several criteria have been found for operators on Fréchet spaces with a continuous norm (see [6], [9], [23], [25], [26] and [27]) and a characterization has even been given for weakly mixing operators on complex Banach spaces by González, León and Montes [18. Moreover, Bès and Conejero [8] have shown that there exist some hypercyclic operators with hypercyclic subspaces on the space $\omega$, where $\omega$ is the space $\mathbb{K}^{\mathbb{Z}_{+}}(\mathbb{K}=\mathbb{R}$ or $\mathbb{C})$ endowed with the product topology. On the other hand, Charpentier, Mouze and the author [16] have obtained the existence of hypercyclic subspaces for universal series on some Fréchet spaces without continuous norm. Nevertheless, no criterion is known in the case of Fréchet spaces without continuous norm.

1991 Mathematics Subject Classification. Primary 47A16.

Key words and phrases. Hypercyclic operators; Hypercyclic subspaces; Fréchet spaces.

The author is supported by a grant of FRIA. 
The starting point of this paper is the following simple remark. There exist two types of hypercyclic subspace $M$ for $\left(T_{k}\right)$ : either there exists a continuous seminorm $p$ on $X$ such that $M \cap \operatorname{ker} p=\{0\}$ (type 1), or for any continuous seminorm $p$ on $X$, the subspace $M \cap \operatorname{ker} p$ is infinite-dimensional (type 2). If $X$ possesses a continuous norm, then each hypercyclic subspace is of type 1 . On the other hand, if each continuous seminorm of $X$ has a kernel of finite codimension, then each hypercyclic subspace is of type 2; this is the case of $\omega$, for example. However, in the other cases, there can exist hypercyclic subspaces of type 1 or of type 2 .

In Section 1, we consider hypercyclic subspaces of type 1. We start by proving the existence of convenient basic sequences in each Fréchet space admitting a continuous seminorm whose kernel is not of finite codimension. Thanks to these basic sequences, we generalize principal criteria about hypercyclic subspaces on Fréchet spaces with a continuous norm. An interesting application of these results concerns the differential operators on the space $C^{\infty}(\mathbb{R})$. We prove in Section 2.1 that for any non-constant polynomial $P$, the operator $P(D)$ possesses a hypercyclic subspace in $C^{\infty}(\mathbb{R})$. We can also prove that, on each Fréchet sequence space admitting a continuous seminorm whose kernel is not of finite codimension, the existence of one restricted universal series implies the existence of a closed infinitedimensional subspace of restricted universal series (Section 2.2). Finally, we answer positively a question posed by Bès and Conejero in [8, Problem 8]: "Does every separable infinite-dimensional Fréchet space support an operator with a hypercyclic subspace?" (Section 2.3).

In Section 3, we focus on hypercyclic subspaces of type 2. We establish a sufficient criterion for having a hypercyclic subspace of type 2 and a sufficient criterion for having no hypercyclic subspace of type 2. These criteria are applied in Section 4 to three classes of hypercyclic operators. We first look at the case of universal series on Fréchet sequence spaces for which each continuous seminorm has a kernel of finite codimension (Section 4.1). In particular, we generalize to these spaces a result obtained for $\omega$ in [16]. This result together with results obtained in Section 2.2 characterizes almost completely the existence of closed infinite-dimensional subspaces of restricted universal series in Fréchet spaces. Afterwards, we improve a result obtained by Bès and Conejero [8] that states that the operators of the form $P\left(B_{w}\right)$, where $P$ is a non-constant polynomial and $B_{w}$ is a weighted shift, possess a hypercyclic subspace on $\omega$; we show that a larger class of sequences of operators from $\omega$ to $\omega$ possesses a hypercyclic subspace and even a frequently hypercyclic subspace (Section 4.2). Finally, we investigate unilateral weighted shifts on Fréchet sequence spaces without continuous norm (Section 4.3).

\section{Some CRITERIA FOR HYPERCYCliC SUbSPACES OF TYPE 1}

We start by proving the simple remark stated in the Introduction.

Proposition 1.1. Let $X$ be a Fréchet space, $\left(p_{n}\right)_{n \geq 1}$ an increasing sequence of seminorms inducing the topology of $X$ and $M$ an infinite-dimensional subspace in $X$. Either for any $n \geq 1, \operatorname{ker} p_{n} \cap M$ is infinite-dimensional or there exists $n \geq 1$ such that $\operatorname{ker} p_{n} \cap M=\{0\}$.

Proof. Suppose that there exists $n \geq 1$ such that $\operatorname{ker} p_{n} \cap M$ is finite-dimensional. Let $e_{1}, \ldots, e_{d}$ be a basis of $\operatorname{ker} p_{n} \cap M$. Since $X$ is a Fréchet space, there exists $m \geq n$ such that $p_{m}\left(e_{1}\right) \neq 0$. Therefore, the dimension of $\operatorname{ker} p_{m} \cap M$ is strictly 
less than $d$. By repeating this argument, we then obtain a seminorm $p_{N}$ such that $\operatorname{ker} p_{N} \cap M=\{0\}$.

In general, the simplest way to obtain a hypercyclic subspace is to construct a convenient basic sequence in $X$ and to consider the closed linear span of this sequence.

Definition 1.2. A sequence $\left(u_{n}\right)_{n \geq 1}$ in a Fréchet space is called basic if for every $x \in \overline{\operatorname{span}}\left\{u_{n}: n \geq 1\right\}$, there exists a unique sequence $\left(a_{n}\right)_{n \geq 1}$ in $\mathbb{K}$ such that $x=\sum_{n=1}^{\infty} a_{n} u_{n}$.

Let $X$ be a Fréchet space. If $X$ possesses a continuous norm, the existence of basic sequences in $X$ is well known (see 24, [27]). We now show that if there exists a continuous seminorm $p$ on $X$ such that ker $p$ is not a subspace of finite codimension, we can generalize the classical construction of basic sequences in Fréchet spaces with a continuous norm to obtain basic sequences $\left(u_{k}\right) \subset X$ such that $p\left(u_{k}\right)=1$ for any $k$ and $\overline{\operatorname{span}}\left\{u_{k}: k \geq 1\right\} \cap \operatorname{ker} p=\{0\}$. Moreover, sufficiently small perturbations of these sequences will remain basic and will be equivalent to the initial sequence.

Definition 1.3. Let $X$ be a Fréchet space. Two basic sequences $\left(u_{n}\right)$ and $\left(f_{n}\right)$ in $X$ are said to be equivalent if for every sequence $\left(a_{n}\right)_{n \geq 1}$ in $\mathbb{K}$, the series $\sum_{n=1}^{\infty} a_{n} u_{n}$ converges in $X$ if and only if $\sum_{n=1}^{\infty} a_{n} f_{n}$ converges in $X$.

These sequences will be the key point to obtain criteria about hypercyclic subspaces of type 1 .

Lemma 1.4 (21, Lemma 10.39]). Let $X$ be a Fréchet space. For any finitedimensional subspace $F$ of $X$, for any continuous seminorm $p$ on $X$ and for any $\varepsilon>0$, there exists a closed subspace $E$ of finite codimension such that for any $x \in E$, for any $y \in F$, we have

$$
p(x+y) \geq \max \left(\frac{p(x)}{2+\varepsilon}, \frac{p(y)}{1+\varepsilon}\right) .
$$

Corollary 1.5. Let $X$ be a Fréchet space, $\left(p_{n}\right)$ a sequence of continuous seminorms and $M$ an infinite-dimensional subspace such that for any closed subspace $E$ of finite codimension, we have

$$
E \cap M \not \subset \operatorname{ker} p_{1} \text {. }
$$

Then for any $\varepsilon>0$, for any $u_{1}, \ldots, u_{n} \in X$, there exists $u_{n+1} \in M$ such that $p_{1}\left(u_{n+1}\right)=1$ and such that for any $j \leq n$, for any $a_{1}, \ldots, a_{n+1} \in \mathbb{K}$, we have

$$
p_{j}\left(\sum_{k=1}^{n} a_{k} u_{k}\right) \leq(1+\varepsilon) p_{j}\left(\sum_{k=1}^{n+1} a_{k} u_{k}\right) .
$$

Remark 1.6. If $\operatorname{ker} p_{1}$ is a subspace of finite codimension, such a subspace $M$ does not exist. However, if $\operatorname{ker} p_{1}$ is not a subspace of finite codimension, then $M=$ $X$ works and if $\operatorname{ker} p_{1}=\{0\}$, then each infinite-dimensional subspace $M$ works. Moreover, we notice that if $M \cap \operatorname{ker} p_{1}=\{0\}$, then $M$ works.

Theorem 1.7. Let $X$ be a Fréchet space, $\left(p_{n}\right)$ an increasing sequence of seminorms defining the topology of $X,\left(\varepsilon_{n}\right)_{n \geq 1}$ a sequence of positive real numbers such that 
$\prod_{n}\left(1+\varepsilon_{n}\right)=K<\infty$. If a sequence $\left(u_{k}\right)_{k \geq 1} \subset X$ satisfies for any $n \in \mathbb{N}$, for any $j \leq n$, for any $a_{1}, \ldots, a_{n+1} \in \mathbb{K}$,

$$
p_{j}\left(\sum_{k=1}^{n} a_{k} u_{k}\right) \leq\left(1+\varepsilon_{n}\right) p_{j}\left(\sum_{k=1}^{n+1} a_{k} u_{k}\right) \quad \text { and } p_{1}\left(u_{n}\right)=1,
$$

then this sequence is basic in $X$ and the closure of the linear span $M_{u}$ of $\left(u_{k}\right)$ satisfies $M_{u} \cap \operatorname{ker} p_{1}=\{0\}$. Moreover, if $\left(f_{k}\right)_{k \geq 1} \subset X$ satisfies

$$
\sum_{k=1}^{+\infty} 2 K p_{k}\left(u_{k}-f_{k}\right)<1
$$

then $\left(f_{k}\right)$ is a basic sequence in $X,\left(f_{k}\right)$ is equivalent to $\left(u_{k}\right)$ and the closure of the linear span $M_{f}$ of $\left(f_{k}\right)$ satisfies $M_{f} \cap \operatorname{ker} p_{1}=\{0\}$.

Proof. We first show that $\left(u_{k}\right)_{k \geq 1}$ is a basic sequence. Let $x=\sum_{k=1}^{\infty} a_{k} u_{k} \in X$. We remark that, by (1.1), we have for any $n \geq 1$

$$
\begin{aligned}
\left|a_{n}\right|=p_{1}\left(a_{n} u_{n}\right) & \leq p_{1}\left(\sum_{k=1}^{n} a_{k} u_{k}\right)+p_{1}\left(\sum_{k=1}^{n-1} a_{k} u_{k}\right) \\
& \leq 2 K p_{1}\left(\sum_{k=1}^{\infty} a_{k} u_{k}\right)=2 K p_{1}(x) .
\end{aligned}
$$

This property implies that if $x=\sum_{k=1}^{\infty} a_{k} u_{k}$ and $x=\sum_{k=1}^{\infty} b_{k} u_{k}$ then for any $n \geq 1, a_{n}=b_{n}$. Let $x_{n}=\sum_{k=1}^{\infty} a_{n, k} u_{k}$, with $a_{n, k}=0$ for any $k \geq N_{n}$, be a convergent sequence to some vector $x \in X$. It just remains to prove that there exists a sequence $\left(a_{k}\right)_{k>1} \subset \mathbb{K}$ such that $x=\sum_{k=1}^{\infty} a_{k} u_{k}$.

We deduce from (1.2) that for any $k \geq 1,\left|a_{m, k}-a_{n, k}\right| \leq 2 K p_{1}\left(x_{m}-x_{n}\right) \rightarrow 0$ as $m, n \rightarrow \infty$ and therefore $\lim _{n \rightarrow \infty} a_{n, k}=a_{k}$ for some scalar $a_{k}$. Let $j, n \geq 1$. For any $N \geq j$, we have by (1.1)

$$
\begin{aligned}
p_{j}\left(\sum_{k=1}^{N} a_{n, k} u_{k}-\sum_{k=1}^{N} a_{k} u_{k}\right) & =\lim _{m} p_{j}\left(\sum_{k=1}^{N} a_{n, k} u_{k}-\sum_{k=1}^{N} a_{m, k} u_{k}\right) \\
& \leq \lim _{m} K p_{j}\left(x_{n}-x_{m}\right)=K p_{j}\left(x_{n}-x\right) .
\end{aligned}
$$

Thus, for any $N \geq \max \left\{N_{n}, j\right\}$, we have

$p_{j}\left(x-\sum_{k=1}^{N} a_{k} u_{k}\right) \leq p_{j}\left(x-x_{n}\right)+p_{j}\left(\sum_{k=1}^{N} a_{n, k} u_{k}-\sum_{k=1}^{N} a_{k} u_{k}\right) \leq(1+K) p_{j}\left(x_{n}-x\right)$.

We deduce that $x=\sum_{k=1}^{\infty} a_{k} u_{k}$ and thus the sequence $\left(u_{k}\right)$ is basic. Moreover, if $M_{u}=\overline{\operatorname{span}}\left\{u_{k}: k \geq 1\right\}$, then for any $x \in M_{u}, x=\sum_{k=1}^{\infty} a_{k} u_{k}$ with $\left|a_{k}\right| \leq 2 K p_{1}(x)$. We conclude that $M_{u} \cap \operatorname{ker} p_{1}=\{0\}$.

We now consider a sequence $\left(f_{k}\right)_{k \geq 1} \subset X$ such that

$$
\delta:=\sum_{k=1}^{+\infty} 2 K p_{k}\left(u_{k}-f_{k}\right)<1 .
$$

In order to prove that $\left(f_{k}\right)$ is a basic sequence, we consider the operator $T: M_{u} \rightarrow X$ given by $T\left(\sum_{n=1}^{\infty} a_{n} u_{n}\right)=\sum_{n=1}^{\infty} a_{n} f_{n}$. This operator is well-defined as for any 
$j \geq 1$, for any $j \leq m \leq n$,

$$
\begin{aligned}
p_{j}\left(\sum_{k=m}^{n} a_{k} f_{k}\right) & \leq p_{j}\left(\sum_{k=m}^{n} a_{k}\left(f_{k}-u_{k}\right)\right)+p_{j}\left(\sum_{k=m}^{n} a_{k} u_{k}\right) \\
& \leq \sum_{k=m}^{n}\left|a_{k}\right| p_{j}\left(f_{k}-u_{k}\right)+p_{j}\left(\sum_{k=m}^{n} a_{k} u_{k}\right) \\
& \leq \sum_{k=m}^{n} 2 K p_{1}\left(\sum_{i=m}^{n} a_{i} u_{i}\right) p_{k}\left(f_{k}-u_{k}\right)+p_{j}\left(\sum_{k=m}^{n} a_{k} u_{k}\right) \quad \text { by (1.2) } \\
& \leq(1+\delta) p_{j}\left(\sum_{k=m}^{n} a_{k} u_{k}\right) \text { by (1.3) }
\end{aligned}
$$

and the operator $T$ is continuous as, for any $j \geq 1$, we have with the same reasoning:

$$
\begin{aligned}
p_{j}\left(T\left(\sum_{k=1}^{\infty} a_{k} u_{k}\right)\right) & \leq \sum_{k=1}^{\infty} 2 K p_{1}\left(\sum_{i=1}^{\infty} a_{i} u_{i}\right) p_{j}\left(f_{k}-u_{k}\right)+p_{j}\left(\sum_{k=1}^{\infty} a_{k} u_{k}\right) \\
& \leq \sum_{k=1}^{j-1} 2 K p_{1}\left(\sum_{i=1}^{\infty} a_{i} u_{i}\right) p_{j}\left(f_{k}-u_{k}\right)+(1+\delta) p_{j}\left(\sum_{k=1}^{\infty} a_{k} u_{k}\right) \\
& \leq\left(1+\delta+\sum_{k=1}^{j-1} 2 K p_{j}\left(f_{k}-u_{k}\right)\right) p_{j}\left(\sum_{k=1}^{\infty} a_{k} u_{k}\right) .
\end{aligned}
$$

We seek to prove that $T$ is an isomorphism between $M_{u}$ and $\operatorname{Im}(T)$. We remark that for any $\sum_{k=1}^{\infty} a_{k} u_{k} \in M_{u}$, for any $j \geq 1$, we have by (1.2) and (1.3)

$$
\begin{aligned}
p_{j}\left(\sum_{k=j}^{\infty} a_{k} f_{k}\right) & \geq p_{j}\left(\sum_{k=j}^{\infty} a_{k} u_{k}\right)-p_{j}\left(\sum_{k=j}^{\infty} a_{k}\left(f_{k}-u_{k}\right)\right) \\
& \geq p_{j}\left(\sum_{k=j}^{\infty} a_{k} u_{k}\right)-\sum_{k=j}^{\infty} 2 K p_{j}\left(\sum_{i=j}^{\infty} a_{i} u_{i}\right) p_{k}\left(f_{k}-u_{k}\right) \\
& \geq p_{j}\left(\sum_{k=j}^{\infty} a_{k} u_{k}\right)-\delta p_{j}\left(\sum_{k=j}^{\infty} a_{k} u_{k}\right) \\
& \geq(1-\delta) p_{j}\left(\sum_{k=j}^{\infty} a_{k} u_{k}\right)
\end{aligned}
$$

Thanks to (1.5) with $j=1$, we can already assert that $T$ is injective, as for any $x \in M_{u} \backslash\{0\}$, we have $p_{1}(x) \neq 0$. We also deduce that $\operatorname{Im}(T) \cap \operatorname{ker} p_{1}=\{0\}$.

Let $x_{n}=\sum_{k=1}^{\infty} a_{n, k} u_{k}$ be a sequence in $M_{u}$ such that $T x_{n}$ converges to $f$ in $X$ as $n \rightarrow \infty$. To prove that $T$ is an isomorphism, we still have to show that $x_{n}$ converges in $X$. Thanks to (1.2) and (1.5), we know that for any $k \geq 1, a_{n, k} \rightarrow a_{k}$ for some scalar $a_{k}$ as $n \rightarrow \infty$. We therefore seek to prove that $x_{n}$ converges to $\sum_{k=1}^{\infty} a_{k} u_{k}$. To this end, we begin by showing that $\sum_{k=1}^{\infty} a_{k} f_{k}$ converges. For any 


$$
\begin{aligned}
N \geq & M \geq j, \text { any } n \geq 1, \text { we have } \\
p_{j}( & \left.\sum_{k=M}^{N} a_{k} f_{k}\right) \\
= & \lim _{m} p_{j}\left(\sum_{k=M}^{N} a_{m, k} f_{k}\right) \\
\leq & \lim _{m} p_{j}\left(\sum_{k=M}^{N} a_{m, k} f_{k}-\sum_{k=M}^{N} a_{n, k} f_{k}\right)+p_{j}\left(\sum_{k=M}^{N} a_{n, k} f_{k}\right) \\
\leq & (1+\delta) \lim _{m} p_{j}\left(\sum_{k=M}^{N}\left(a_{m, k}-a_{n, k}\right) u_{k}\right)+p_{j}\left(\sum_{k=M}^{N} a_{n, k} f_{k}\right) \quad \text { by (1.4) } \\
\leq & 2 K(1+\delta) \limsup _{m} p_{j}\left(\sum_{k=j}^{\infty}\left(a_{m, k}-a_{n, k}\right) u_{k}\right)+p_{j}\left(\sum_{k=M}^{N} a_{n, k} f_{k}\right) \quad \text { by (1.1) } \\
\leq & \frac{2 K(1+\delta)}{1-\delta} \limsup _{m} p_{j}\left(\sum_{k=j}^{\infty}\left(a_{m, k}-a_{n, k}\right) f_{k}\right)+p_{j}\left(\sum_{k=M}^{N} a_{n, k} f_{k}\right) \quad \text { by (1.5) } \\
\leq & \frac{2 K(1+\delta)}{1-\delta} \limsup _{m} p_{j}\left(T x_{m}-T x_{n}\right) \\
& +\frac{2 K(1+\delta)}{1-\delta} \limsup _{m} p_{j}\left(\sum_{k=1}^{j-1} a_{m, k} f_{k}-\sum_{k=1}^{j-1} a_{n, k} f_{k}\right)+p_{j}\left(\sum_{k=M}^{N} a_{n, k} f_{k}\right) \\
\leq & \frac{2 K(1+\delta)}{1-\delta} p_{j}\left(f-T x_{n}\right) \\
& +\frac{2 K(1+\delta)}{1-\delta} p_{j}\left(\sum_{k=1}^{j-1} a_{k} f_{k}-\sum_{k=1}^{j-1} a_{n, k} f_{k}\right)+p_{j}\left(\sum_{k=M}^{N} a_{n, k} f_{k}\right) .
\end{aligned}
$$

If we choose $n \geq 1$ such that

$$
\frac{2 K(1+\delta)}{1-\delta} p_{j}\left(f-T x_{n}\right)+\frac{2 K(1+\delta)}{1-\delta} p_{j}\left(\sum_{k=1}^{j-1} a_{k} f_{k}-\sum_{k=1}^{j-1} a_{n, k} f_{k}\right)<\varepsilon
$$

and $L \geq j$ such that, for any $N \geq M \geq L$, we have

$$
p_{j}\left(\sum_{k=M}^{N} a_{n, k} f_{k}\right)<\varepsilon,
$$

then we deduce that, for any $N \geq M \geq L$, we have

$$
p_{j}\left(\sum_{k=M}^{N} a_{k} f_{k}\right) \leq 2 \varepsilon
$$

The sequence $\left(\sum_{k=1}^{N} a_{k} f_{k}\right)_{N}$ is thus a Cauchy sequence and therefore a convergent sequence. Moreover, as for any $N \geq M \geq j$, we have by (1.5)

$$
p_{j}\left(\sum_{k=M}^{N} a_{k} u_{k}\right) \leq \frac{1}{1-\delta} p_{j}\left(\sum_{k=M}^{N} a_{k} f_{k}\right)
$$


we deduce that the sequence $\left(\sum_{k=1}^{N} a_{k} u_{k}\right)$ is also a Cauchy sequence and therefore $\sum_{k=1}^{\infty} a_{k} u_{k}$ converges.

We can now show that $f=\sum_{k=1}^{\infty} a_{k} f_{k}$. Indeed, since $\sum_{k=1}^{\infty} a_{k} f_{k}$ and $\sum_{k=1}^{\infty} a_{n, k} f_{k}$ are convergent series, for any $n, j \geq 1$, there exists $M \geq j$ such that we have

$$
p_{j}\left(\sum_{k=M+1}^{\infty} a_{k} f_{k}-\sum_{k=M+1}^{\infty} a_{n, k} f_{k}\right)<\frac{1}{n}
$$

and thus as previously

$$
\begin{aligned}
p_{j}\left(\sum_{k=j}^{\infty} a_{k} f_{k}-\sum_{k=j}^{\infty} a_{n, k} f_{k}\right) \leq & \frac{1}{n}+p_{j}\left(\sum_{k=j}^{M} a_{k} f_{k}-\sum_{k=j}^{M} a_{n, k} f_{k}\right) \\
= & \frac{1}{n}+\lim _{m} p_{j}\left(\sum_{k=j}^{M} a_{m, k} f_{k}-\sum_{k=j}^{M} a_{n, k} f_{k}\right) \\
\leq & \frac{1}{n}+\frac{2 K(1+\delta)}{1-\delta} p_{j}\left(f-T x_{n}\right) \\
& +\frac{2 K(1+\delta)}{1-\delta} p_{j}\left(\sum_{k=1}^{j-1} a_{k} f_{k}-\sum_{k=1}^{j-1} a_{n, k} f_{k}\right) \stackrel{n \rightarrow \infty}{\longrightarrow} 0 .
\end{aligned}
$$

We conclude that $T x_{n} \rightarrow \sum_{k=1}^{\infty} a_{k} f_{k}$ as $n \rightarrow \infty$ and thus that $f=\sum_{k=1}^{\infty} a_{k} f_{k}$.

Finally, we notice that $x_{n}$ converges to $\sum_{k=1}^{\infty} a_{k} u_{k}$ because by (1.5), for any $j \geq 1$

$$
\begin{aligned}
& p_{j}\left(\sum_{k=1}^{\infty} a_{k} u_{k}-x_{n}\right) \\
& \quad \leq p_{j}\left(\sum_{k=1}^{j-1} a_{k} u_{k}-\sum_{k=1}^{j-1} a_{n, k} u_{k}\right)+p_{j}\left(\sum_{k=j}^{\infty} a_{k} u_{k}-\sum_{k=j}^{\infty} a_{n, k} u_{k}\right) \\
& \quad \leq p_{j}\left(\sum_{k=1}^{j-1} a_{k} u_{k}-\sum_{k=1}^{j-1} a_{n, k} u_{k}\right)+\frac{1}{1-\delta} p_{j}\left(\sum_{k=j}^{\infty} a_{k} f_{k}-\sum_{k=j}^{\infty} a_{n, k} f_{k}\right) \stackrel{n \rightarrow \infty}{\longrightarrow} 0 .
\end{aligned}
$$

We conclude that $T$ is an isomorphism between $M_{u}$ and $\operatorname{Im}(T)$. The subspace $M^{\prime}:=\operatorname{Im}(T)$ is thus a closed infinite-dimensional subspace for which $\left(f_{k}\right)$ is a basis. Moreover, we have $M^{\prime} \cap \operatorname{ker} p_{1}=\{0\}$ and $\left(f_{k}\right)$ is equivalent to $\left(u_{k}\right)$. That concludes the proof.

We deduce from Corollary 1.5] and Theorem 1.7 that we can construct a basic sequence stable under small perturbations in each infinite-dimensional subspace $M \subset X$ such that for any closed subspace $E$ of finite codimension,

$$
E \cap M \not \subset \operatorname{ker} p_{1} \text {. }
$$

If $M$ is closed, we can in fact reformulate this condition as follows:

Proposition 1.8. Let $X$ be a Fréchet space, $p$ a continuous seminorm on $X$ and $M$ a closed infinite-dimensional subspace of $X$. The following assertions are equivalent:

(1) for any closed subspace $E$ of finite codimension in $X, E \cap M \not \subset \operatorname{ker} p$,

(2) the subspace $M \cap \operatorname{ker} p$ is of infinite codimension in $M$. 
Proof. We show the equivalence between $\neg(1)$ and $\neg(2)$.

$\neg(1) \Rightarrow \neg(2)$. Let $E \subset X$ be a closed subspace of finite codimension such that $E \cap M \subset \operatorname{ker} p$. Then the subspace $E \cap M$ is a subspace of finite codimension in $M$ and since $E \cap M \subset M \cap \operatorname{ker} p \subset M$, we deduce that $M \cap \operatorname{ker} p$ is of finite codimension in $M$.

$\neg(2) \Rightarrow \neg(1)$. We suppose that $M \cap \operatorname{ker} p$ is of finite codimension in $M$. Since $M \cap \operatorname{ker} p$ is closed, there exists $n \geq 1$ and a continuous linear map $f: M \rightarrow \mathbb{K}^{n}$ such that ker $f=M \cap \operatorname{ker} p$. By Hahn-Banach, there then exists a continuous linear map $\tilde{f}: X \rightarrow \mathbb{K}^{n}$ such that $\tilde{f}_{\mid M}=f$. We conclude that $E=\operatorname{ker} \tilde{f}$ is a closed subspace of finite codimension and that

$$
E \cap M \subset \operatorname{ker} f \subset \operatorname{ker} p .
$$

Thanks to Theorem 1.7 we can extend known criteria about hypercyclic subspaces for Fréchet spaces with a continuous norm to Fréchet spaces without continuous norm by adapting their statement.

The first criterion about hypercyclic subspaces was established by Montes [26] in 1996. He has proved that if $T$ is a continuous linear operator on a Banach space $X$ satisfying the Hypercyclicity Criterion for $\left(n_{k}\right)$ and if there exists a closed infinitedimensional subspace $M_{0}$ such that for any $x \in M_{0},\left(T^{n_{k}} x\right)_{k}$ converges to 0 , then $T$ possesses a hypercyclic subspace. This criterion has then been generalized to operators on Fréchet spaces with a continuous norm (see [9], 27]) and to sequences of operators on separable Banach spaces 23 and on separable Fréchet spaces with a continuous norm [25] satisfying a certain condition $(C)$. An elegant alternative proof of this criterion via left-multiplication operators has also been obtained by Chan [14].

Definition 1.9. Let $X$ be a Fréchet space and $Y$ a topological vector space. A sequence $\left(T_{n}\right)$ of operators from $X$ to $Y$ satisfies condition $(\mathrm{C})$ if there exist an increasing sequence $\left(n_{k}\right)$ of positive integers and a dense subset $X_{0} \subset X$ such that

(1) for every $x \in X_{0}, \lim _{k \rightarrow \infty} T_{n_{k}} x=0$;

(2) for every continuous seminorm $p$ on $X, \bigcup_{k} T_{n_{k}}(\{x \in X: p(x)<1\})$ is dense in $Y$.

One knows that the above mentioned criterion does not remain true for Fréchet spaces without continuous norm. Indeed, Bonet, Martínez and Peris 9] have shown that the multiple of the bilateral backward shift $2 B$ is mixing on the space $X=$ $\left\{\left(x_{n}\right)_{n \in \mathbb{Z}}: \sum_{n=0}^{\infty}\left|x_{n}\right|<\infty\right\}$ endowed with the seminorms $p_{n}(x)=\sum_{k=-n}^{\infty}\left|x_{k}\right|$ and does not possess any hypercyclic subspace. However, if we consider $M_{0}=$ $\left\{\left(x_{n}\right)_{n \in \mathbb{Z}}: x_{n}=0\right.$ for $\left.n \geq 1\right\}$, we remark that for any $x \in M_{0},\left((2 B)^{k} x\right)_{k}$ converges to 0 as $k \rightarrow \infty$. In fact, the problem in this counterexample does not seem to be the absence of a continuous norm on $X$ but the fact that $M_{0} \cap \operatorname{ker} p_{n}$ is a subspace of finite codimension in $M_{0}$ for any $n \geq 1$. Nevertheless, once $M_{0} \cap \operatorname{ker} p_{1}$ is a subspace of infinite codimension in $M_{0}$, the construction of basic sequences of Theorem 1.7 allows us to generalize the criterion established by Montes [26] to Fréchet spaces without continuous norm.

We fix a Fréchet space $X$, an increasing sequence of seminorms $\left(p_{n}\right)$ defining the topology of $X$ and a separable topological vector space $Y$ whose topology is defined 
by a sequence of seminorms $\left(q_{n}\right)$. We can then state the following result whose the proof is similar to the proof of [25, Theorem 1.5].

Theorem 1.10. Let $\left(T_{n}\right)$ be a sequence of continuous linear operators from $X$ to $Y$. If $\left(T_{n}\right)$ satisfies condition $(C)$ for $\left(n_{k}\right)$ and if there exists a closed infinitedimensional subspace $M_{0}$ of $X$ such that

(1) $M_{0} \cap \operatorname{ker} p_{1}$ is a subspace of infinite codimension in $M_{0}$,

(2) for any $x \in M_{0}, T_{n_{k}} x \rightarrow 0$ as $k \rightarrow \infty$, then $\left(T_{n}\right)$ possesses a hypercyclic subspace of type 1 .

Remark 1.11. A simple argument (see [6], [21, Remark 10.9]) likewise allows us to replace (2) by the condition: for any $x \in M_{0},\left(T_{n_{k}} x\right)_{k}$ converges in $Y$.

León and Müller 23] have established another sufficient condition for sequences of operators on Banach spaces to have a hypercyclic subspace. This criterion relies on the existence of a convenient non-increasing sequence of infinite-dimensional closed subspaces $\left(M_{j}\right)$. It has also been generalized to the case of Fréchet spaces with a continuous norm in [25]. By adding a condition on the subspaces $\left(M_{j}\right)$, we can extend this criterion to Fréchet spaces without continuous norm:

Theorem 1.12. Let $\left(T_{n}\right)$ be a sequence of continuous linear operators from $X$ to $Y$. If $\left(T_{n}\right)$ satisfies condition $(C)$ for $\left(n_{k}\right)$ and if there exists a non-increasing sequence of infinite-dimensional closed subspaces $\left(M_{j}\right)_{j \geq 1}$ of $X$ such that

(1) for any $j \geq 1, M_{j} \cap \operatorname{ker} p_{1}$ is a subspace of infinite codimension in $M_{j}$,

(2) for each $n \geq 1$, there exist a positive number $C_{n}$ and two integers $m(n)$, $k(n) \geq 1$ such that for any $j \geq k(n)$, for any $x \in M_{j}$,

$$
q_{n}\left(T_{n_{j}} x\right) \leq C_{n} p_{m(n)}(x),
$$

then $\left(T_{n}\right)$ possesses a hypercyclic subspace of type 1.

On the other hand, a criterion for having no hypercylic subspace is given in 23 . for sequences of operators on Banach spaces. It has been adapted for operators on Fréchet spaces in 21] and for sequences of operators on Fréchet spaces in 25]. These results directly give us a condition for having no hypercyclic subspaces of type 1 .

Theorem 1.13. Let $\left(T_{n}\right)$ be a sequence of continuous linear operators from $X$ to $Y$. If there exist a sequence $\left(E_{n}\right)$ of closed subspaces of finite codimension in $X$, positive numbers $C_{n} \rightarrow \infty$ and a continuous seminorm $q$ on $Y$ such that for any $n \geq 1$, for any $x \in E_{n}$, we have

$$
q\left(T_{n} x\right) \geq C_{n} p_{n}(x)
$$

then $\left(T_{n}\right)$ does not possess any hypercyclic subspace of type 1 .

Proof. Suppose that $M$ is a hypercyclic subspace of type 1. By definition, there then exists $J \geq 1$ such that for any non-zero vector $x \in M$, we have $p_{J}(x) \neq 0$. Therefore, the proof of [25, Theorem 1.13] allows us to construct a vector $x \in M$ such that $q\left(T^{n} x\right) \rightarrow \infty$ as $n \rightarrow \infty$ : this is a contradiction with the fact that $M$ is a hypercyclic subspace.

Thanks to a simplification of the previous criterion in the case of an operator $T: X \rightarrow X$ given by the author in [25], we obtain the following simpler criterion: 
Theorem 1.14. Let $T$ be a continuous linear operator from $X$ to itself. If there exists a continuous seminorm $q$ on $X$ such that for any $n \geq 1$, there exist a closed subspace $E$ of finite codimension in $X, C>1$ and $m \geq 1$ such that for any $x \in E$,

$$
q\left(T^{m} x\right) \geq C p_{n}(x)
$$

then $T$ does not possess any hypercyclic subspace of type 1 .

\section{EXISTENCE OF HYPERCYCLIC SUBSPACES}

2.1. Differential operators on $C^{\infty}(\mathbb{R})$. Let $C^{\infty}(\mathbb{R})$ be the Fréchet space of infinitely differentiable functions on $\mathbb{R}$ endowed with the increasing sequence of seminorms

$$
p_{k}(f):=\sum_{l=0}^{k-1} \sup _{x \in[-k, k]}\left|f^{(l)}(x)\right|,
$$

where we denote by $f^{(l)}$ the $l$ th derivative of $f$. The space $C^{\infty}(\mathbb{R})$ is an important example of Fréchet spaces without continuous norm and one can wonder if the differential operators possess hypercyclic subspaces on this space.

In the case of the space of entire functions $H(\mathbb{C})$, we know that each differential operator $\varphi(D)$, where $D$ is the derivative operator and $\varphi$ is a non-constant entire function of exponential type, possesses a hypercyclic subspace in $H(\mathbb{C})$. This result has been proved by Petersson [27] for entire functions which are not polynomials, by Shkarin [29] for the operator $D$ and completed by the author 25] in the case of non-constant polynomials. In the case of the space $C^{\infty}(\mathbb{R})$, we remark that the map $\varphi(D)$ will be a well-defined operator on $C^{\infty}(\mathbb{R})$ if and only if $\varphi$ is a polynomial. Indeed, by Borel theorem [13], we know that for any sequence $\left(a_{n}\right)_{n \geq 0} \in \mathbb{R}^{\mathbb{Z}_{+}}$, there exists a function $f \in C^{\infty}(\mathbb{R})$ such that for any $n \geq 0, f^{(n)}(0)=a_{n}$.

Let $P$ be a non-constant polynomial. We already know thanks to Godefroy and Shapiro [17] that the operator $P(D)$ is hypercyclic on $C^{\infty}(\mathbb{R})$. We now show that for every non-constant polynomial $P$, the operator $P(D)$ possesses a hypercyclic subspace of type 1 in $C^{\infty}(\mathbb{R})$.

Lemma 2.1. Let $T$ be an operator on $C^{\infty}(\mathbb{R})$. If for any $k \geq 1$,

$$
T\left(\operatorname{ker} p_{k}\right) \subset \operatorname{ker} p_{k},
$$

then for any closed subspace $E$ of finite codimension in $C^{\infty}(\mathbb{R})$, any $\varepsilon>0$, any $N \geq 1$, there exists $f \in E$ such that $p_{1}(f)=1$ and for any $1 \leq l, k \leq N$,

$$
p_{k}\left(T^{l} f\right) \leq \varepsilon p_{k+1}(f) .
$$

Proof. Let $E$ be a closed subspace of finite codimension in $C^{\infty}(\mathbb{R})$ and $d$ the codimension of $E$. Let $\varepsilon>0$ and $N \geq 1$. We let $\left.I_{j, k}=\right] k+\frac{j}{d+1}, k+\frac{j+1}{d+1}[$ for any $0 \leq j \leq d$, any $0 \leq k \leq N$. For any $0 \leq j \leq d$, we then choose $f_{j, 0}, \ldots, f_{j, N} \in C^{\infty}(\mathbb{R})$ such that

- for any $0 \leq k \leq N, \operatorname{supp}\left(f_{j, k}\right) \subset I_{j, k}$;

- $p_{1}\left(f_{j, 0}\right)=1$

- for any $1 \leq l, k \leq N$,

$$
p_{k}\left(T^{l}\left(\sum_{i=0}^{k-1} f_{j, i}\right)\right) \leq \varepsilon \sup _{x \in I_{j, k}}\left|f_{j, k}(x)\right| .
$$


Let $f_{j}=\sum_{k=0}^{N} f_{j, k}, j=0, \ldots, d$. Since for any $k \geq 1, T\left(\operatorname{ker} p_{k}\right) \subset \operatorname{ker} p_{k}$ and $f_{j, k} \in \operatorname{ker} p_{k}$, we deduce that for any $0 \leq j \leq d$, any $1 \leq l, k \leq N$,

$$
\begin{aligned}
p_{k}\left(T^{l} f_{j}\right)=p_{k}\left(T^{l}\left(\sum_{i=0}^{k-1} f_{j, i}\right)\right) & \leq \varepsilon \sup _{x \in I_{j, k}}\left|f_{j, k}(x)\right| \\
& \leq \varepsilon \sup _{x \in[-k-1, k+1]}\left|f_{j}(x)\right| \leq \varepsilon p_{k+1}\left(f_{j}\right) .
\end{aligned}
$$

Since $E$ is a subspace of finite codimension $d$ and $f_{0}, \ldots, f_{d}$ are clearly linearly independent, there exists $a_{0}, \ldots, a_{d}$ with $\max \left\{\left|a_{j}\right|: 0 \leq j \leq d\right\}=1$ such that $f:=a_{0} f_{0}+\cdots+a_{d} f_{d} \in E$. Since the supports of the $f_{j}$ are disjoint, we have

$$
p_{1}(f)=\max _{0 \leq j \leq d}\left|a_{j}\right| p_{1}\left(f_{j}\right)=\max _{0 \leq j \leq d}\left|a_{j}\right| p_{1}\left(f_{j, 0}\right)=1 .
$$

Moreover, we deduce from (2.1) that for any $1 \leq l, k \leq N$,

$$
\begin{aligned}
p_{k}\left(T^{l} f\right) & \leq \sum_{j=0}^{d}\left|a_{j}\right| p_{k}\left(T^{l} f_{j}\right) \leq \varepsilon \sum_{j=0}^{d}\left|a_{j}\right| p_{k+1}\left(f_{j}\right) \\
& \leq(d+1) \varepsilon \max _{0 \leq j \leq d}\left|a_{j}\right| p_{k+1}\left(f_{j}\right) \leq(d+1) \varepsilon p_{k+1}(f) .
\end{aligned}
$$

Theorem 2.2. Let $T$ be an operator on $C^{\infty}(\mathbb{R})$. If $T$ satisfies condition $(C)$ and if for any $k \geq 1, T\left(\operatorname{ker} p_{k}\right) \subset \operatorname{ker} p_{k}$, then $T$ possesses a hypercyclic subspace of type 1.

Proof. Let $\left(n_{k}\right)_{k \geq 1}$ be an increasing sequence of positive integers such that $T$ satisfies condition $(C)$ for $\left(n_{k}\right)$ and $\left(\varepsilon_{n}\right)_{n \geq 1}$ a sequence of positive real numbers such that $\prod_{n}\left(1+\varepsilon_{n}\right) \leq 2$. Thanks to Lemma 1.4 and Lemma 2.1 we know that there exists a sequence $\left(u_{k}\right)_{k \geq 1} \subset C^{\infty}(\mathbb{R})$ satisfying (1.1) for the sequence $\left(\varepsilon_{n}\right)_{n \geq 1}$ and such that for any $1 \leq j, n \leq k$,

$$
p_{n}\left(T^{n_{j}} u_{k}\right) \leq \frac{1}{2^{k}} p_{n+1}\left(u_{k}\right) .
$$

We show that $T$ satisfies the assumptions of Theorem 1.12 for the sequence of closed infinite-dimensional subspaces $M_{j}=\overline{\operatorname{span}}\left\{u_{k}: k \geq j\right\}, m(n)=n+1, k(n)=n+2$ and $C_{n}=1$.

By Theorem 1.7, we know that $\overline{\operatorname{span}}\left\{u_{k}: k \geq 1\right\} \cap$ ker $p_{1}=\{0\}$. The subspace $M_{j} \cap \operatorname{ker} p_{1}$ is thus of infinite codimension in $M_{j}$. On the other hand, since $\left(u_{n}\right)_{n \geq 1}$ is a basic sequence (Theorem 1.7), we know that for any $x \in M_{j}$, we have $x=$ $\sum_{k=j}^{\infty} a_{k} u_{k}$ for some sequence $\left(a_{k}\right)_{k \geq 1} \in \mathbb{K}^{\mathbb{N}}$ and we deduce from (1.1) and (2.2) that for any $n \geq 1$, any $j \geq n+2$, any $x \in M_{j}, x=\sum_{k=j}^{\infty} a_{k} u_{k}$, we have

$$
\begin{aligned}
p_{n}\left(T^{n_{j}} x\right) & \leq \sum_{k=j}^{\infty} p_{n}\left(T^{n_{j}}\left(a_{k} u_{k}\right)\right) \leq \sum_{k=j}^{\infty} \frac{1}{2^{k}} p_{n+1}\left(a_{k} u_{k}\right) \\
& \leq \sum_{k=j}^{\infty} \frac{4}{2^{k}} p_{n+1}(x) \leq p_{n+1}(x) .
\end{aligned}
$$

Corollary 2.3. For any non-constant polynomial $P$, the operator $P(D)$ possesses a hypercyclic subspace of type 1 in $C^{\infty}(\mathbb{R})$. 
Proof. Let $P$ be a non-constant polynomial. We know that the operator $P(D)$ satisfies the Hypercyclicity Criterion [17]. Therefore, since for any $k \geq 1$,

$$
P(D)\left(\operatorname{ker} p_{k}\right) \subset \operatorname{ker} p_{k},
$$

we deduce from Theorem 2.2 that $P(D)$ possesses a hypercyclic subspace of type 1 in $C^{\infty}(\mathbb{R})$.

Remark 2.4. For any non-constant polynomial $P$, the operator $P(D)$ does not possess any hypercyclic subspace of type 2 in $C^{\infty}(\mathbb{R})$ since there is no hypercyclic vector for $P(D)$ in $\operatorname{ker} p_{1}$.

2.2. Universal series. Let $A$ be a Fréchet space of sequences such that the coordinate projections $P_{m}: A \rightarrow \mathbb{K},\left(a_{n}\right)_{n \geq 0} \mapsto a_{m}$ are continuous for all $m \geq 0$, and the set of polynomials $\left\{a=\left(a_{n}\right)_{n \geq 0} \in \mathbb{K}^{\mathbb{Z}_{+}}:\left\{n \geq 0: a_{n} \neq 0\right\}\right.$ is finite $\}$ is contained and dense in $A$. We denote by $\left(e_{n}\right)_{n \geq 0}$ the canonical basis of $\mathbb{K}^{\mathbb{Z}_{+}}$and by $v(a)$ and $d(a)$ the valuation and the degree of the polynomial $a \in A$.

Let $X$ be a separable topological vector space whose topology is given by an increasing sequence of seminorms $\left(q_{j}\right)$ and let $\left(x_{n}\right)_{n \geq 0}$ be a sequence in $X$. We let $S_{k}: A \rightarrow X$ be the operator defined by

$$
S_{k}\left(\left(a_{n}\right)_{n \geq 0}\right)=\sum_{n=0}^{k} a_{n} x_{n}
$$

and $\mathcal{U} \cap A$ the set of hypercyclic vectors for $\left(S_{k}\right)$. A sequence $a \in \mathcal{U} \cap A$ is called a universal series. An interesting subclass of universal series is the class of restricted universal series.

Definition 2.5. Let $S_{k}^{A}: A \rightarrow A$ be the operator defined by

$$
S_{k}^{A}\left(\left(a_{n}\right)_{n \geq 0}\right)=\sum_{n=0}^{k} a_{n} e_{n} .
$$

A sequence $a \in A$ is a restricted universal series if, for every $x \in X$, there exists an increasing sequence $\left(n_{k}\right)$ in $\mathbb{N}$ such that

$$
S_{n_{k}} a \rightarrow x \text { in } X \text { and } S_{n_{k}}^{A} a \rightarrow a \text { in } A \text { as } k \rightarrow \infty \text {. }
$$

We denote by $\mathcal{U}_{A}$ the set of such series.

For more details about universal series, we refer to the article of Bayart, GrosseErdmann, Nestoridis and Papadimitropoulos [4.

In 2010, Charpentier [15] proved that if $\mathcal{U}_{A} \neq \emptyset$ and $A$ is a Banach space then $\mathcal{U}_{A}$ is spaceable i.e. $\mathcal{U}_{A} \cup\{0\}$ contains a closed infinite-dimensional subspace. Using the construction of basic sequences in Fréchet spaces with a continuous norm, the author [24] has generalized this result to Fréchet spaces with a continuous norm. The proof of these results depends on three factors: the existence of a restricted universal series, the possibility to construct a basic sequence of polynomials with valuation as large as desired and the fact that a sufficiently small perturbation of this basic sequence remains basic. We prove that, thanks to Theorem 1.7, these conditions are satisfied in the case where $\mathcal{U}_{A} \neq \emptyset$ and $A$ is a Fréchet space admitting a continuous seminorm $p$ such that ker $p$ is not a subspace of finite codimension. 
Theorem 2.6. If $\mathcal{U}_{A}$ is non-empty and $A$ is a Fréchet space with a continuous seminorm $p$ such that $\operatorname{ker} p$ is not a subspace of finite codimension, then $\mathcal{U}_{A}$ is spaceable.

Proof. The proof is similar to the proof for Fréchet spaces with a continuous norm 24. Therefore, we only justify the possibility to construct a basic sequence of polynomials with valuation as large as desired thanks to Corollary 1.5] and Theorem 1.7. To this end, we prove that the subspace $M_{k}$ of polynomials with valuation at least $k$ satisfies

$E \cap M_{k} \not \subset \operatorname{ker} p \quad$ for any closed subspace $E$ of finite codimension.

Since $<e_{0}, \ldots, e_{k-1}>\oplus M_{k}$ is dense, we have

$$
A=\overline{<e_{0}, \ldots, e_{k-1}>\oplus M_{k}}=<e_{0}, \ldots, e_{k-1}>\oplus \overline{M_{k}} .
$$

The subspace $\overline{M_{k}}$ is thus of finite codimension in $A$. Let $E$ be a closed subspace of finite codimension in $X$. There exists a finite-dimensional subspace $F$ such that $\left(E \cap M_{k}\right) \oplus F=M_{k}$. We deduce that

$$
\overline{M_{k}}=\overline{\left(E \cap M_{k}\right) \oplus F}=\overline{\left(E \cap M_{k}\right)} \oplus F .
$$

Since $\overline{M_{k}}$ is a subspace of finite codimension in $A$, we conclude that $\overline{E \cap M_{k}}$ is a subspace of finite codimension in $A$ and thus that we cannot have $E \cap M_{k} \subset \operatorname{ker} p$ as ker $p$ is not a subspace of finite codimension.

2.3. Existence of mixing operators with hypercyclic subspaces. The existence of hypercyclic and even mixing operators on any infinite-dimensional separable Fréchet space is now well known. In fact, the existence of hypercyclic operators has been obtained on any infinite-dimensional separable Banach space by Ansari [1] and Bernal [5], and on any infinite-dimensional separable Fréchet space by Bonet and Peris 10. The construction of these operators is based on the hypercyclity of perturbations of the identity by a weighted shift. Grivaux has then noticed in [19] that these operators are even mixing.

In 1997, León and Montes [22] proved that every infinite-dimensional separable Banach space supports an operator with a hypercyclic subspace. In 2006, this result was generalized for infinite-dimensional separable Fréchet spaces with a continuous norm independently by Bernal [6] and Petersson [27]. For Fréchet spaces isomorphic to $\omega$, the same result was obtained by Bès and Conejero [8]. Theorem 1.10 permits us to answer positively the question asked in [8, Problem 8]: "Does every separable infinite-dimensional Fréchet space support an operator with a hypercyclic subspace?".

Lemma $2.7([10])$. Let $X$ be a separable infinite-dimensional Fréchet space. If $X$ is not isomorphic to $\omega$ then there exists a dense subspace $F$ of $X$ which has a continuous norm $p$ and there are sequences $\left(x_{n}\right)_{n} \subset F$ and $\left(f_{n}\right)_{n} \subset X^{\prime}$, the dual of $X$, such that

(1) $\left(x_{n}\right)_{n}$ converges to 0 , and $\operatorname{span}\left\{x_{n}: n \in \mathbb{N}\right\}$ is dense in $X$;

(2) $\left(f_{n}\right)_{n}$ is $X$-equicontinuous in $X^{\prime}$;

(3) $\left\langle x_{n}, f_{m}\right\rangle=0$ if $n \neq m$ and $\left.\left(\left\langle x_{n}, f_{n}\right\rangle\right)_{n} \subset\right] 0,1[$.

Theorem 2.8. Every separable infinite-dimensional Fréchet space supports a mixing operator with a hypercyclic subspace. 
Proof. Let $B: \omega \rightarrow \omega$ be the backward shift. Since the operator $B$ is a mixing operator with a hypercyclic subspace in $\omega$ (see [8]), any space $X$ isomorphic to $\omega$ supports a mixing operator with a hypercyclic subspace.

Now, if $X$ is not isomorphic to $\omega$, then Lemma 2.7 permits us to construct a mixing operator $T: X \rightarrow X$ (see [10, 19]) defined by

$$
T x=x+\sum_{n=1}^{\infty} 2^{-n}\left\langle x, f_{2 n}\right\rangle x_{n} .
$$

In particular, since $T$ is mixing, $T$ satisfies the Hypercyclicity Criterion and thus condition $(C)$.

Let $F$ and $p$ as given by Lemma 2.7. There exists a continuous seminorm $q$ on $X$ such that the restriction of $q$ to $F$ coincides with $p$. The subspace $M_{0}:=\overline{\operatorname{span}}\left\{x_{n}\right.$ : $n$ odd $\}$ then satisfies the assumptions of Theorem 1.10 with the modification introduced in Remark 1.11, since each vector in $M_{0}$ is a fixed point and

$$
\operatorname{span}\left\{x_{n}: n \text { odd }\right\} \cap \operatorname{ker} q=\{0\} .
$$

We conclude by Theorem 1.10 .

\section{SOME CRITERIA FOR HYPERCYCLIC SUBSPACES OF TYPE 2}

We move from our study of hypercyclic subspaces of type 1 to the study of hypercyclic subspaces of type 2 . In order to construct hypercyclic subspaces of type 2 , we first have to find a way to construct basic sequences $\left(u_{n}\right)$ in $X$ such that if we denote by $M_{u}$ the closed linear span of $\left(u_{n}\right)$, then $M_{u} \cap \operatorname{ker} p$ is infinitedimensional for any continuous seminorm $p$ on $X$.

Lemma 3.1. Let $X$ be a Fréchet space, $\left(p_{n}\right)$ an increasing sequence of seminorms inducing the topology of $X$ and $\left(u_{n}\right)_{n \geq 1} \subset X$. If for any $n \geq 1, u_{n} \in \operatorname{ker} p_{n} \backslash \operatorname{ker} p_{n+1}$, then $\left(u_{n}\right)$ is a basic sequence,

$$
M_{u}:=\overline{\operatorname{span}}\left\{u_{n}: n \geq 1\right\}=\left\{\sum_{n=1}^{\infty} \alpha_{n} u_{n}:\left(\alpha_{n}\right) \in \mathbb{K}^{\mathbb{N}}\right\}
$$

and for any $k \geq 1$,

$$
M_{u} \cap \operatorname{ker} p_{k}=\overline{\operatorname{span}}\left\{u_{n}: n \geq k\right\}=\left\{\sum_{n=k}^{\infty} \alpha_{n} u_{n}:\left(\alpha_{n}\right) \in \mathbb{K}^{\mathbb{N}}\right\} .
$$

In particular, the space $M_{u} \cap \operatorname{ker} p$ is infinite-dimensional for any continuous seminorm $p$ on $X$.

Proof. Let $\left(u_{n}\right)$ be a sequence in $X$ such that for any $n \geq 1, u_{n} \in \operatorname{ker} p_{n} \backslash \operatorname{ker} p_{n+1}$. For any sequence $\left(\alpha_{n}\right) \in \mathbb{K}^{\mathbb{N}}$, the series $\sum_{n=1}^{\infty} \alpha_{n} u_{n}$ converges because for any $k \geq 1$, any $M \geq N \geq k$, we have $p_{k}\left(\sum_{n=N}^{M} \alpha_{n} u_{n}\right)=0$. Moreover, if $x=\sum_{n=1}^{\infty} \alpha_{n} u_{n}$ and $x=\sum_{n=1}^{\infty} \beta_{n} u_{n}$, then for any $n \geq 1, \alpha_{n}=\beta_{n}$. Indeed, suppose that $n_{0}$ is the smallest index such that $\alpha_{n_{0}} \neq \beta_{n_{0}}$, then we get the following contradiction:

$$
0=p_{n_{0}+1}\left(\sum_{n=1}^{\infty} \alpha_{n} u_{n}-\sum_{n=1}^{\infty} \beta_{n} u_{n}\right)=\left|\alpha_{n_{0}}-\beta_{n_{0}}\right| p_{n_{0}+1}\left(u_{n_{0}}\right) \neq 0 .
$$

Let $x \in X$ and $x_{k}=\sum_{n=1}^{\infty} \alpha_{n, k} u_{n}$. To finish the proof, it suffices to show that if the sequence $\left(x_{k}\right)_{k}$ converges to $x$ in $X$, then each sequence $\left(\alpha_{n, k}\right)_{k}$ converges to some scalar $\alpha_{n}$ and $x=\sum_{n=1}^{\infty} \alpha_{n} u_{n}$. We first notice that since $p_{2}\left(x_{k}-x_{j}\right)=$ 
$\left|\alpha_{1, k}-\alpha_{1, j}\right| p_{2}\left(u_{1}\right)$ converges to 0 as $k, j \rightarrow \infty$, the sequence $\left(\alpha_{1, k}\right)_{k}$ is a Cauchy sequence and thus converges to some scalar $\alpha_{1}$. Then we remark by induction that for any $N \geq 2$, the sequence $\left(\alpha_{N, k}\right)_{k}$ is a Cauchy sequence and thus converges to some scalar $\alpha_{N}$. Therefore, we have, for any $N \geq 2$,

$$
\begin{aligned}
p_{N}\left(x-\sum_{n=1}^{\infty} \alpha_{n} u_{n}\right) & \leq p_{N}\left(x-x_{k}\right)+p_{N}\left(x_{k}-\sum_{n=1}^{\infty} \alpha_{n} u_{n}\right) \\
& =p_{N}\left(x-x_{k}\right)+p_{N}\left(\sum_{n=1}^{N-1} \alpha_{n, k} u_{n}-\sum_{n=1}^{N-1} \alpha_{n} u_{n}\right) \stackrel{k \rightarrow \infty}{\longrightarrow} 0 .
\end{aligned}
$$

Remark 3.2. Such a basic sequence does not remain basic under small perturbations; for example in $\omega,\left(e_{k}\right)_{k \geq 0}$ is a basic sequence but $\left(e_{k}+\varepsilon_{k} e_{k-1}\right)_{k \geq 0}$, with $e_{-1}=0$, is not basic for any $\varepsilon_{k}>0$.

Let $\left(T_{k}\right)$ be a sequence of linear operators from $X$ to $Y$, where $X$ is an infinitedimensional Fréchet space without continuous norm, whose topology is given by an increasing sequence of seminorms $\left(p_{n}\right)$, and where $Y$ is a separable topological vector space, whose topology is given by an increasing sequence of seminorms $\left(q_{j}\right)$. Using the previous construction of basic sequences, we can state a sufficient criterion for the existence of hypercyclic subspaces of type 2 .

Theorem 3.3. If there exist an increasing sequence $\left(n_{k}\right)$ and a set $X_{0} \subset X$ such that

1. for any $j \geq 1$, for any $x \in X_{0},\left(q_{j}\left(T_{n_{k}} x\right)\right)_{k}$ is ultimately zero;

2. for any $n, K \geq 1, \bigcup_{k \geq K} T_{n_{k}}\left(X_{0} \cap \operatorname{ker} p_{n}\right)$ is dense in $Y$,

then $\left(T_{n}\right)$ possesses a hypercyclic subspace of type 2 .

Proof. If for any continuous seminorm $q$ on $Y$, for any $y \in Y, q(y)=0$, then $X$ is a hypercyclic subspace of type 2 because, by our assumption, $X$ does not admit a continuous norm. If this is not the case, there exists a dense sequence $\left(y_{l}\right)_{l \geq 1}$ in $Y$ such that for any $l \geq 1, q_{j}\left(y_{l}\right) \neq 0$ for some $j \geq 1$. Without loss of generality, we can suppose that $q_{l}\left(y_{l}\right) \neq 0$. By continuity of $T_{n_{k}}$, we also notice that for any $K \geq 1$, there exists $N_{K} \geq 1$ and $C>0$ such that for any $x \in X$,

$$
\max _{k \leq K} q_{k}\left(T_{n_{k}} x\right) \leq C p_{N_{K}}(x) .
$$

In order to use Lemma 3.1, we then seek to construct a sequence $\left(u_{k}\right) \subset X$ such that for any $k \geq 1, u_{k} \in \operatorname{ker} p_{k} \backslash \operatorname{ker} p_{k+1}$ and for any $\left(\alpha_{k}\right)_{k} \subset \mathbb{K}^{\mathbb{N}} \backslash\{0\}$, the series $\sum_{k=1}^{\infty} \alpha_{k} u_{k}$ is hypercyclic for $\left(T_{n_{k}}\right)_{k}$. By hypothesis, for any $\varepsilon>0$, for any $l, K, n \geq 1$, there exist $x \in X_{0} \cap \operatorname{ker} p_{n}$ and $k \geq K$ such that

$$
q_{l}\left(T_{n_{k}} x-y_{l}\right)<\varepsilon
$$

and since $q_{l}\left(y_{l}\right) \neq 0$, we can assume that $x \neq 0$ so that there exists $m>n$ such that $p_{m}(x) \neq 0$. For any $l, n, K_{0} \geq 1$, there then exist $x \in X_{0} \cap \operatorname{ker} p_{n}$ and an increasing sequence $\left(K_{j}\right)_{j \geq 1}$ such that

- for any $i \leq K_{0}, \quad q_{i}\left(T_{n_{i}} x\right)=0\left(\operatorname{choosing} x \in \operatorname{ker} p_{N_{K_{0}}}\right)$;

- there exists $K_{0} \leq i<K_{1}$, such that $q_{l}\left(T_{n_{i}} x-y_{l}\right)<\frac{1}{l}$;

- for any $j \geq 1$, any $i \geq K_{j}, q_{j}\left(T_{n_{i}} x\right)=0$ (because $\left.x \in X_{0}\right)$. 
We construct recursively a family $\left(x_{k, l}\right)_{k, l \geq 1} \subset X_{0}$ and a family $\left(n_{\phi(k, l)}\right)_{k, l \geq 1}$, for the strict order $\prec$ defined by $\left(k^{\prime}, l^{\prime}\right) \prec(k, l)$ if $k^{\prime}+l^{\prime}<k+l$ or if $k^{\prime}+l^{\prime}=k+l$ and $l^{\prime}<l$, such that

- for any $k \geq 1,(\phi(k, l))_{l}$ is strictly increasing;

- for any $k, l \geq 1, x_{k, l} \in \operatorname{ker} p_{k+l}$ and $q_{l}\left(T_{n_{\phi(k, l)}} x_{k, l}-y_{l}\right)<\frac{1}{l}$;

- for any $k, l \geq 1$, there exists an increasing sequence $\left(K_{j}^{k, l}\right)_{j \geq 0}$ such that

(a) $K_{0}^{k, l} \geq l$

(b) $\phi(k, l) \in\left[K_{0}^{k, l}, K_{1}^{k, l}[\right.$;

(c) for each pair $\left(k^{\prime}, l^{\prime}\right) \prec(k, l), K_{l}^{k^{\prime}, l^{\prime}}<K_{0}^{k, l}$;

(d) for any $i \leq K_{0}^{k, l}, q_{i}\left(T_{n_{i}} x_{k, l}\right)=0$;

(e) for any $j \geq 1$, any $i \geq K_{j}^{k, l}, q_{j}\left(T_{n_{i}} x_{k, l}\right)=0$.

Since we use the seminorm $p_{k+2}$ only after the construction of $x_{k, 1}$, we may assume that $p_{k+2}\left(x_{k, 1}\right) \neq 0$, by changing the seminorm $p_{k+2}$ if necessary.

Let $u_{k}=\sum_{l=1}^{\infty} x_{k, l}$ which clearly converges. We therefore remark that we have $p_{k+1}\left(u_{k}\right)=0$ and $p_{k+2}\left(u_{k}\right) \neq 0$, and thus by Lemma 3.1

$$
M:=\overline{\operatorname{span}}\left\{u_{k}: k \geq 1\right\}=\left\{\sum_{k=1}^{\infty} \alpha_{k} u_{k}:\left(\alpha_{k}\right) \in \mathbb{K}^{\mathbb{N}}\right\} .
$$

Moreover, by our construction, we notice that for any $l_{0} \geq 1$ :

- if $(k, l) \prec\left(k_{0}, l_{0}\right)$ then, by (b) and (c), we have $K_{l_{0}}^{k, l}<K_{0}^{k_{0}, l_{0}} \leq \phi\left(k_{0}, l_{0}\right)$ and thus, by (e), $q_{l_{0}}\left(T_{n_{\phi\left(k_{0}, l_{0}\right)}} x_{k, l}\right)=0$;

- if $\left(k_{0}, l_{0}\right) \prec(k, l)$ then, by (a), (b) and (c), we have

$$
l_{0} \leq K_{0}^{k_{0}, l_{0}} \leq \phi\left(k_{0}, l_{0}\right)<K_{l}^{k_{0}, l_{0}}<K_{0}^{k, l}
$$

and thus, by (d), $q_{l_{0}}\left(T_{n_{\phi\left(k_{0}, l_{0}\right)}} x_{k, l}\right) \leq q_{\phi\left(k_{0}, l_{0}\right)}\left(T_{n_{\phi\left(k_{0}, l_{0}\right)}} x_{k, l}\right)=0$.

Therefore, if $u=\sum_{k=1}^{\infty} \alpha_{k} u_{k}$ with $\alpha_{k_{0}}=1$, we have for any $l_{0} \geq 1$ :

$$
q_{l_{0}}\left(T_{n_{\phi\left(k_{0}, l_{0}\right)}} u-y_{l_{0}}\right)=q_{l_{0}}\left(T_{n_{\phi\left(k_{0}, l_{0}\right)}} x_{k_{0}, l_{0}}-y_{l_{0}}\right)<\frac{1}{l_{0}} .
$$

We conclude that each non-zero vector in $M$ is hypercyclic and thus that $M$ is a hypercyclic subspace of type 2 .

Example 3.4. Every non-constant translation operator on $C^{\infty}(\mathbb{R})$ possesses a hypercyclic subspace of type 2. This result directly follows from Theorem 3.3 by considering the set of infinitely differentiable functions with finite support.

We can simplify the previous criterion if each continuous seminorm on $X$ has a kernel of finite codimension.

Corollary 3.5. Suppose that each seminorm $p_{n}$ has a kernel of finite codimension. If there exist an increasing sequence $\left(n_{k}\right)$ and a set $X_{0} \subset X$ such that

1. for any $j \geq 1$, any $x \in X_{0},\left(q_{j}\left(T_{n_{k}} x\right)\right)_{k}$ is ultimately zero;

2. for any $K \geq 1, \bigcup_{k \geq K} T_{n_{k}}\left(X_{0}\right)$ is dense in $Y$.

then $\left(T_{n}\right)$ possesses a hypercyclic subspace.

Proof. Thanks to Theorem 3.3, it is sufficient to prove that for any $n, K \geq 1$, for any non-empty open set $U \subset Y$, there exists $x \in \operatorname{ker} p_{n}$ such that

(1) for any $j \geq 1,\left(q_{j}\left(T_{n_{k}} x\right)\right)_{k}$ is ultimately zero; 
(2) for some $k \geq K, T_{n_{k}} x \in U$.

Let $n, K \geq 1$ and $U$ a non-empty open set in $Y$. We denote by $d$ the codimension of ker $p_{n}$. If for any continuous seminorm $q$ on $Y$, for any $y \in Y, q(y)=0$, then $X$ is a hypercyclic subspace of type 2 . If this is not the case, each non-empty open set in $Y$ contains a vector $y$ satisfying $q(y) \neq 0$ for some continuous seminorm $q$ on $Y$ and there thus exist $y_{0} \in Y, j_{0} \geq 1$ and $\varepsilon>0$ such that

$$
\left\{y \in Y: q_{j_{0}}\left(y_{0}-y\right)<\varepsilon\right\} \subset U \text { and } \varepsilon<q_{j_{0}}\left(y_{0}\right) .
$$

By hypothesis, there exists $x_{1} \in X_{0}$ such that for some $k_{1} \geq K$,

$$
T_{n_{k_{1}}} x_{1} \in\left\{y \in Y: q_{j_{0}}\left(y_{0}-y\right)<\varepsilon\right\} .
$$

Since $x_{1} \in X_{0}$, there also exists $K_{1} \geq k_{1}$ such that for any $k \geq K_{1}, q_{j_{0}}\left(T_{n_{k}} x_{1}\right)=0$. In particular, for any $k \geq K_{1}, T_{n_{k}} x_{1} \notin\left\{y \in Y: q_{j_{0}}\left(y_{0}-y\right)<\varepsilon\right\}$. Therefore, since $\bigcup_{k \geq K_{1}} T_{n_{k}}\left(X_{0}\right)$ is dense in $X$, there exists another vector $x_{2} \in X_{0}$ such that for some $k_{2} \geq K_{1}$,

$$
T_{n_{k_{2}}} x_{2} \in\left\{y \in Y: q_{j_{0}}\left(y_{0}-y\right)<\varepsilon\right\} .
$$

We can thus find $x_{1}, \ldots, x_{d+1} \in X_{0}$ and $k_{1} \leq \cdots \leq k_{d+1}$ such that for any $1 \leq$ $i \leq d+1, T_{n_{k_{i}}} x_{i} \in U$ and for any $1 \leq i<j \leq d+1, q_{j_{0}}\left(T_{n_{k_{j}}} x_{i}\right)=0$. In particular, $x_{1}, \ldots, x_{d+1}$ are linearly independent because for any $1 \leq j \leq d+1$, any $a_{1}, \ldots, a_{j} \in \mathbb{K}$ with $a_{j} \neq 0$,

$$
q_{j_{0}}\left(T_{n_{k_{j}}}\left(a_{1} x_{1}+\cdots+a_{j} x_{j}\right)\right)=\left|a_{j}\right| q_{j_{0}}\left(T_{n_{k_{j}}} x_{j}\right) \neq 0 .
$$

Since ker $p_{n}$ has codimension $d$, we deduce that there exist $a_{1}, \ldots, a_{d+1}$ such that $x:=a_{1} x_{1}+\cdots+a_{d+1} x_{d+1} \in \operatorname{ker} p_{n} \backslash\{0\}$. Therefore, since $x_{1}, \ldots, x_{d+1} \in X_{0}$, $\left(q_{j}\left(T_{n_{k}} x\right)\right)_{k}$ is ultimately zero for any $j \geq 1$ and if we let $i_{0}=\max \{1 \leq i \leq d+1$ : $\left.a_{i} \neq 0\right\}$, without loss of generality $a_{i_{0}}=1$ and $T_{n_{k_{i}}} x \in U$. The result follows.

Now we establish a sufficient criterion for not having a hypercyclic subspace of type 2 .

Theorem 3.6. Let $X_{j}=\left\{x \in X: \#\left\{k: q_{j}\left(T_{k} x\right)=0\right\}=\infty\right\}$. If there exist $j, N, K \geq 1$ and a subspace $E$ of finite codimension in $X$ such that

$$
\bigcup_{k \geq K} T_{k}\left(\operatorname{ker} p_{N} \cap E \cap X_{j}\right) \quad \text { is not dense in } Y \text {, }
$$

then $\left(T_{k}\right)$ does not possess any hypercyclic subspace of type 2 .

Proof. By hypothesis, there exist a non-empty open set $U \subset Y, j, N, K \geq 1$ and a subspace of finite codimension $E$ in $X$ such that

$$
\left(\bigcup_{k \geq K} T_{k}\left(\operatorname{ker} p_{N} \cap E \cap X_{j}\right)\right) \cap U=\emptyset .
$$

A direct consequence is that for any $x \in \operatorname{ker} p_{N} \cap E$, if there exists $k \geq K$ such that $T_{k} x \in U$, then $\left(q_{j}\left(T_{k} x\right)\right)$ is ultimately non-zero. Suppose that there exists a hypercyclic subspace $M$ of type 2 . Then we can consider a sequence $\left(u_{n}\right)_{n \geq 1}$ of non-zero vectors such that for any $n \geq 1, u_{n} \in M \cap \operatorname{ker} p_{n+N} \cap E$. In particular, for any $n \geq 1, u_{n}$ is hypercyclic and there thus exists $k_{n} \geq K$ such that $T_{k_{n}} u_{n} \in U$. Since $u_{n} \in \operatorname{ker} p_{N} \cap E$, we deduce by our previous reasoning that there exists $K_{n} \geq 1$ such that for any $k>K_{n}$,

$$
q_{j}\left(T_{k} u_{n}\right) \neq 0 .
$$


Q. MENET

We seek to construct a sequence $\left(\alpha_{n}\right)_{n \geq 1} \in \mathbb{R}^{\mathbb{N}}$ such that if we let $u=\sum_{n=1}^{\infty} \alpha_{n} u_{n}$, which exists because $u_{n} \in \operatorname{ker} p_{n+N}$, then, for any $k>K_{1}$, we have $q_{j}\left(T_{k}(u)\right) \geq 1$. That will give us the desired contradiction as $u \in M \backslash\{0\}$ and $u$ is not hypercyclic. To this end, we start by choosing $\alpha_{1}$ such that for any $K_{1}<k \leq K_{2}$, we have

$$
q_{j}\left(\alpha_{1} T_{k} u_{1}\right)>1
$$

Then we choose $\alpha_{n}$ recursively such that for any $K_{1}<k \leq K_{n+1}$, we have

$$
q_{j}\left(T_{k}\left(\sum_{l=1}^{n-1} \alpha_{l} u_{l}\right)+\alpha_{n} T_{k} u_{n}\right)>1 \text {. }
$$

Such a choice exists because for any $K_{n}<k \leq K_{n+1}$, we have $q_{j}\left(T_{k}\left(u_{n}\right)\right) \neq 0$ and for any $K_{1}<k \leq K_{n}$, either we have $q_{j}\left(T_{k}\left(u_{n}\right)\right) \neq 0$ and we have just to choose $\alpha_{n}$ sufficiently large, or we have $q_{j}\left(T_{k}\left(u_{n}\right)\right)=0$ and we have the desired inequality by the induction hypothesis. We conclude by continuity of $\left(T_{k}\right)$ and $q_{j}$.

\section{Some EXAmples of HyPERCyCliC Subspaces OF TyPE 2}

4.1. Universal series. We refer to Section 2.2 for notations and definitions about universal series. We only recall that $A$ is a Fréchet space of sequences whose topology is given by an increasing sequence of seminorms $\left(p_{n}\right)_{n \geq 1}, X$ is a separable topological vector space whose topology is given by an increasing sequence of seminorms $\left(q_{j}\right)_{j \geq 1},\left(x_{n}\right)_{n \geq 0}$ is a sequence in $X$ and $S_{k}: A \rightarrow X$ is the operator defined by

$$
S_{k}\left(\left(a_{n}\right)_{n \geq 0}\right)=\sum_{n=0}^{k} a_{n} x_{n}
$$

Thanks to the criteria of Section 3, we obtain the following two results:

Theorem 4.1. Suppose that for any $n \geq 1$, ker $p_{n}$ is a subspace of finite codimension. If for any $N \geq 0$,

$$
\bigcup_{M \geq N}\left(\operatorname{span}\left\{x_{k}: N \leq k \leq M\right\} \cap \operatorname{span}\left\{x_{k}: M+1 \leq k\right\}\right)
$$

is dense in $X$, then the sequence $\left(S_{k}\right)$ possesses a hypercyclic subspace.

Proof. Let $y \in X, N \geq 0$ and $l \geq 1$. There exist $N \leq M<L$ and $a_{N}, \ldots, a_{L} \in \mathbb{K}$ such that

$$
q_{l}\left(\sum_{k=N}^{M} a_{k} x_{k}-y\right)<\frac{1}{l} \text { and } \sum_{k=N}^{M} a_{k} x_{k}=\sum_{k=M+1}^{L} a_{k} x_{k} .
$$

Let $a^{(y, N, l)}=\sum_{k=N}^{M} a_{k} e_{k}-\sum_{k=M+1}^{L} a_{k} e_{k}$. We then have $q_{l}\left(S_{M} a^{(y, N, l)}-y\right)<\frac{1}{l}$ and $S_{k} a^{(y, N, l)}=0$ for any $k \geq L$. We deduce that the conditions of Corollary 3.5 are satisfied for $X_{0}=\left\{a^{(y, N, l)}: y \in X, N \geq 0, l \geq 1\right\}$.

Theorem 4.2. Suppose that for any $n \geq 1$, $\operatorname{ker} p_{n}$ is a subspace of finite codimension. If there exist $N \geq 0$ and $j \geq 1$ such that

$$
\bigcup_{M \geq N}\left(\operatorname{span}\left\{x_{k}: N \leq k \leq M\right\} \cap\left(\operatorname{span}\left\{x_{k}: M+1 \leq k\right\}+\operatorname{ker} q_{j}\right)\right)
$$

is not dense in $X$, then the sequence $\left(S_{k}\right)$ does not possess any hypercyclic subspace. 
Proof. Let $N \geq 0, j \geq 1$ such that

$$
\bigcup_{M \geq N}\left(\operatorname{span}\left\{x_{k}: N \leq k \leq M\right\} \cap\left(\operatorname{span}\left\{x_{k}: M+1 \leq k\right\}+\operatorname{ker} q_{j}\right)\right)
$$

is not dense in $X$. Let $X_{j}=\left\{a \in A: \#\left\{k: q_{j}\left(S_{k} a\right)=0\right\}=\infty\right\}$. We remark that

$$
\begin{aligned}
\bigcup_{M \geq N} S_{M}\left(\overline{\operatorname{span}}\left\{e_{k}: k \geq N\right\} \cap X_{j}\right) \\
\quad=\bigcup_{M \geq N}\left(\operatorname{span}\left\{x_{k}: N \leq k \leq M\right\} \cap\left(\operatorname{span}\left\{x_{k}: M+1 \leq k\right\}+\operatorname{ker} q_{j}\right)\right) .
\end{aligned}
$$

We conclude by Theorem 3.6 .

In the case where $X$ possesses a continuous norm, we obtain the following generalization of the characterization given by Charpentier, Mouze and the author for the Fréchet space $\omega$ in [16].

Corollary 4.3. Suppose that $X$ possesses a continuous norm and for any $n \geq 1$, $\operatorname{ker} p_{n}$ is a subspace of finite codimension. Then the sequence $\left(S_{k}\right)$ possesses a hypercyclic subspace if and only if for any $N \geq 0$,

$$
\bigcup_{M \geq N}\left(\operatorname{span}\left\{x_{k}: N \leq k \leq M\right\} \cap \operatorname{span}\left\{x_{k}: M+1 \leq k\right\}\right)
$$

is dense in $X$.

With a suitable adaptation of previous ideas, we can modify the condition of Theorem 4.1 to know when $\mathcal{U}_{A}$ is spaceable. We recall that we denote by $S_{k}^{A}: A \rightarrow A$ the operator defined by

$$
S_{k}^{A}\left(\left(a_{n}\right)_{n \geq 0}\right)=\sum_{n=0}^{k} a_{n} e_{n} .
$$

Theorem 4.4. Suppose that for any $n \geq 1, \operatorname{ker} p_{n}$ is a subspace of finite codimension. If for any $N \geq 0$, any $\varepsilon>0$,

$$
\begin{aligned}
\bigcup_{L>M \geq N}\left(\left\{\sum_{k=N}^{M} a_{k} x_{k}: p_{N}\left(\sum_{k=N}^{M} a_{k} e_{k}\right)<\varepsilon\right\}\right. \\
\left.\cap\left\{\sum_{k=M+1}^{L} a_{k} x_{k}: p_{N}\left(\sum_{k=M+1}^{L} a_{k} e_{k}\right)<\varepsilon\right\}\right)
\end{aligned}
$$

is dense in $X$, then $\mathcal{U}_{A}$ is spaceable.

Proof. Let $U$ be a non-empty open set in $X, N \geq 0$ and $\varepsilon>0$. By hypothesis, there exist a polynomial $a=\sum_{k=N}^{L} a_{k} e_{k} \in A$ and $N \leq M<L$ such that

$$
p_{N}(a)<\varepsilon, p_{N}\left(a-S_{M}^{A} a\right)<\varepsilon, S_{M} a \in U \text { and } S_{L} a=0 .
$$

Since $\operatorname{ker} p_{N}$ is a subspace of finite codimension, there even exists such a polynomial $a$ such that $p_{N}(a)=0$. Indeed, if we let $d$ be the codimension of ker $p_{N}$, we know that there exist polynomials $s_{1}, \ldots, s_{d+1} \in A$ such that for any $1 \leq j \leq d+1$, for some $v\left(s_{j}\right) \leq M_{j}<d\left(s_{j}\right)$,

$$
p_{N}\left(s_{j}\right)<\varepsilon, p_{N}\left(s_{j}-S_{M_{j}}^{A} s_{j}\right)<\varepsilon, S_{M_{j}} s_{j} \in U \text { and } S_{d\left(s_{j}\right)} s_{j}=0
$$


and such that additionally $d\left(s_{j}\right)<v\left(s_{j+1}\right)$ for any $1 \leq j \leq d$. There then exist $\lambda_{1}, \ldots, \lambda_{d+1} \in \mathbb{K}$ such that $\lambda_{1} s_{1}+\cdots+\lambda_{d+1} s_{d+1} \in \operatorname{ker} p_{N} \backslash\{0\}$. Moreover, since we can suppose that $\max \left|\lambda_{i}\right| \leq 1$ and $\lambda_{k}=1$ for some $1 \leq k \leq d+1$, we deduce that for any $N \geq 1$, any $\varepsilon>0$ and for any non-empty open set $U$, we can construct a non-zero polynomial $a$ with valuation as large as desired such that for some $v(a) \leq M<d(a)$

$$
p_{N}(a)=0, p_{N}\left(a-S_{M}^{A} a\right)<\varepsilon, S_{M} a \in U \text { and } S_{d(a)} a=0 .
$$

Let $\left(y_{l}\right)_{l>1}$ be a dense sequence in $X$. We construct recursively a family of polynomials $\left(a^{k, l}\right)_{k, l \geq 1}$, for the strict order $\prec$ defined by $\left(k^{\prime}, l^{\prime}\right) \prec(k, l)$ if $k^{\prime}+l^{\prime}<$ $k+l$ or if $k^{\prime}+l^{\prime}=k+l$ and $l^{\prime}<l$, such that

- for any $\left(k^{\prime}, l^{\prime}\right) \prec(k, l), d\left(a^{k^{\prime}, l^{\prime}}\right)<v\left(a^{k, l}\right)$;

- for any $k, l \geq 1$, there exists $v\left(a^{k, l}\right) \leq n_{k, l}<d\left(a^{k, l}\right)$ such that

$$
p_{k+l}\left(a^{k, l}\right)=0, p_{l}\left(a^{k, l}-S_{n_{k, l}}^{A} a^{k, l}\right)<\frac{1}{l}, q_{l}\left(S_{n_{k, l}} a^{k, l}-y_{l}\right)<\frac{1}{l} \text { and } S_{d\left(a^{k, l}\right)} a^{k, l}=0 ;
$$

- for any $k \geq 1, p_{k+2}\left(a^{k, 1}\right) \neq 0$ (changing $p_{k+2}$ if necessary).

We can change $p_{k+2}$ during the construction because we consider this one only after the choice of $a^{k, 1}$.

Let $a^{(k)}=\sum_{l=1}^{\infty} a^{k, l}$. We remark that we have $p_{k+1}\left(a^{(k)}\right)=0$ and $p_{k+2}\left(a^{(k)}\right) \neq 0$, and thus by Lemma 3.1 that

$$
\overline{\operatorname{span}}\left\{a^{(k)}: k \geq 1\right\}=\left\{\sum_{k=1}^{\infty} \alpha_{k} a^{(k)}:\left(\alpha_{k}\right) \in \mathbb{K}^{\mathbb{N}}\right\} .
$$

Therefore, if $a=\sum_{k=1}^{\infty} \alpha_{k} a^{(k)}$ with $\alpha_{k_{0}}=1$, we deduce that for any $l_{0} \geq 1$,

$$
q_{l_{0}}\left(S_{n_{k_{0}, l_{0}}} a-y_{l_{0}}\right)=q_{l_{0}}\left(S_{n_{k_{0}, l_{0}}} a^{k_{0}, l_{0}}-y_{l_{0}}\right)<\frac{1}{l_{0}} \underset{l_{0} \rightarrow \infty}{\longrightarrow} 0
$$

and since $(k, l) \succ\left(k_{0}, l_{0}\right)$ implies $k+l \geq l_{0}$,

$$
\begin{aligned}
p_{l_{0}}\left(a-S_{n_{k_{0}, l_{0}}}^{A} a\right) & =p_{l_{0}}\left(a^{k_{0}, l_{0}}-S_{n_{k_{0}, l_{0}}}^{A} a^{k_{0}, l_{0}}+\sum_{(k, l) \succ\left(k_{0}, l_{0}\right)} \alpha_{k} a^{k, l}\right) \\
& =p_{l_{0}}\left(a^{k_{0}, l_{0}}-S_{n_{k_{0}, l_{0}}}^{A} a^{k_{0}, l_{0}}\right)<\frac{1}{l_{0}} \underset{l_{0} \rightarrow \infty}{\longrightarrow} 0 .
\end{aligned}
$$

We conclude that $\overline{\operatorname{span}}\left\{a^{(k)}: k \geq 1\right\} \subset \mathcal{U}_{A} \cup\{0\}$ and thus $\mathcal{U}_{A}$ is spaceable.

Theorem 4.5. Suppose that for any $n \geq 1$, $\operatorname{ker} p_{n}$ is a subspace of finite codimension. If there exist $N \geq 0, j \geq 1$ and $\varepsilon>0$ such that

$$
\begin{aligned}
\bigcup_{L>M \geq N}\left(\left\{\sum_{k=N}^{M} a_{k} x_{k}: p_{N}\left(\sum_{k=N}^{M} a_{k} e_{k}\right)<\varepsilon\right\}\right. \\
\left.\cap\left(\left\{\sum_{k=M+1}^{L} a_{k} x_{k}: p_{N}\left(\sum_{k=M+1}^{L} a_{k} e_{k}\right)<\varepsilon\right\}+\operatorname{ker} q_{j}\right)\right)
\end{aligned}
$$

is not dense in $X$, then $\mathcal{U}_{A}$ is not spaceable. 
Proof. Let $N \geq 0, j \geq 1, \varepsilon>0$ and $U \subset X$ a non-empty open set such that $U$ does not intersect

$$
\begin{aligned}
\bigcup_{L>M \geq N}\left(\left\{\sum_{k=N}^{M} a_{k} x_{k}:\right.\right. & \left.p_{N}\left(\sum_{k=N}^{M} a_{k} e_{k}\right)<\varepsilon\right\} \\
& \left.\cap\left(\left\{\sum_{k=M+1}^{L} a_{k} x_{k}: p_{N}\left(\sum_{k=M+1}^{L} a_{k} e_{k}\right)<\varepsilon\right\}+\operatorname{ker} q_{j}\right)\right) .
\end{aligned}
$$

That means that for every sequence $a \in A$ with $v(a) \geq N$, if there exist $v(a) \leq M<L$ such that $p_{N}\left(S_{M}^{A} a\right)<\varepsilon, p_{N}\left(S_{L}^{A} a-S_{M}^{A} a\right)<\varepsilon$ and $S_{M} a \in U$, then $q_{j}\left(S_{L} a\right) \neq 0$.

Suppose that $\mathcal{U}_{A} \cup\{0\}$ contains a closed infinite-dimensional subspace $M_{h}$. For any $n \geq 1$, since $\operatorname{ker} p_{N+n}$ is a subspace of finite codimension, there exits a non-zero sequence $a^{(n)} \in M_{h} \cap \operatorname{ker} p_{N+n}$ with valuation $v\left(a^{(n)}\right) \geq N$. Since $a^{(n)} \in \mathcal{U}_{A}$, there also exists an integer $K_{n} \geq N+n$ such that $K_{n} \geq v\left(a^{(n)}\right)$ and

$$
S_{K_{n}} a^{(n)} \in U \text { and } p_{N}\left(S_{K_{n}}^{A} a^{(n)}\right)<\frac{\varepsilon}{2} .
$$

Thanks to the properties of $K_{n}$, we get that for any $n \geq 1$, any $k>K_{n}$, if $p_{N}\left(S_{k}^{A} a^{(n)}\right) \leq \frac{\varepsilon}{2}$, then $p_{N}\left(S_{k}^{A} a^{(n)}-S_{K_{n}}^{A} a^{(n)}\right)<\varepsilon$ and thus by properties of $N, j, \varepsilon, U$, we have $q_{j}\left(S_{k} a^{(n)}\right) \neq 0$. We deduce that for any $k>K_{n}$, we have

$$
q_{j}\left(S_{k} a^{(n)}\right) \neq 0 \text { or } p_{N}\left(S_{k}^{A} a^{(n)}\right)>\frac{\varepsilon}{2} .
$$

We seek to construct $a=\sum_{n=1}^{\infty} \alpha_{n} a^{(n)} \in M_{h}$ such that for any $k>K_{1}$, we have $q_{j}\left(S_{k} a\right) \geq 1$ or $p_{N}\left(a-S_{k}^{A} a\right) \geq \frac{\varepsilon}{2}$. That will contradict the fact that each nonzero vector in $M_{h}$ is a restricted universal series. We remark that since for any $n \geq 1, a^{(n)} \in \operatorname{ker} p_{N+n}$, the series $\sum_{n=1}^{\infty} \alpha_{n} a^{(n)}$ converges in $X$ for every sequence $\left(\alpha_{n}\right) \in \mathbb{K}^{\mathbb{N}}$, and since $M_{h}$ is a closed subspace, $\sum_{n=1}^{\infty} \alpha_{n} a^{(n)} \in M_{h}$. Moreover, if $a=\sum_{n=1}^{\infty} \alpha_{n} a^{(n)}$, we have $p_{N}\left(a-S_{k}^{A} a\right)=p_{N}\left(S_{k}^{A} a\right)$. It thus suffices to construct a sequence $\left(\alpha_{n}\right) \in \mathbb{K}^{\mathbb{N}}$ such that for any $k>K_{1}$, we have

$$
q_{j}\left(S_{k} a\right) \geq 1 \text { or } p_{N}\left(S_{k}^{A} a\right) \geq \frac{\varepsilon}{2} .
$$

We thus begin by choosing $\alpha_{1}$ such that for any $K_{1}<k \leq K_{2}$, we have

$$
q_{j}\left(\alpha_{1} S_{k} a^{(1)}\right)>1 \text { or } p_{N}\left(\alpha_{1} S_{k}^{A} a^{(1)}\right)>\frac{\varepsilon}{2} .
$$

Then, we choose $\alpha_{n}$ recursively such that for any $K_{1}<k \leq K_{n+1}$, we have

$$
q_{j}\left(S_{k}\left(\sum_{k=1}^{n-1} \alpha_{k} a^{(k)}\right)+\alpha_{n} S_{k} a^{(n)}\right)>1 \text { or } p_{N}\left(S_{k}^{A}\left(\sum_{k=1}^{n-1} \alpha_{k} a^{(k)}\right)+\alpha_{n} S_{k}^{A} a^{(n)}\right)>\frac{\varepsilon}{2} \text {. }
$$

Such a choice exists because for any $K_{n}<k \leq K_{n+1}$, we have either $q_{j}\left(S_{k}\left(a^{(n)}\right)\right) \neq$ 0 or $p_{N}\left(S_{k}^{A} a^{(n)}\right)>\frac{\varepsilon}{2}$ and, for any $K_{1}<k \leq K_{n}$, if we have $q_{j}\left(S_{k} a^{(n)}\right) \neq 0$ or $p_{N}\left(S_{k}^{A} a^{(n)}\right) \neq 0$, then it suffices to choose $\alpha_{n}$ sufficiently large and if we have $q_{j}\left(S_{k} a^{(n)}\right)=0$ and $p_{N}\left(S_{k}^{A} a^{(n)}\right)=0$, then we have the desired result by the induction hypothesis.

Corollary 4.6. Suppose that $X$ possesses a continuous norm and for any $n \geq 1$, $\operatorname{ker} p_{n}$ is a subspace of finite codimension. Then the space $\mathcal{U}_{A}$ is spaceable if and 
only if for any $N \geq 0$, any $\varepsilon>0$,

$$
\begin{aligned}
\bigcup_{L>M \geq N}\left(\left\{\sum_{k=N}^{M} a_{k} x_{k}: p_{N}\left(\sum_{k=N}^{M} a_{k} e_{k}\right)<\varepsilon\right\}\right. \\
\left.\cap\left\{\sum_{k=M+1}^{L} a_{k} x_{k}: p_{N}\left(\sum_{k=M+1}^{L} a_{k} e_{k}\right)<\varepsilon\right\}\right)
\end{aligned}
$$

is dense in $X$.

This Corollary together with Theorem 2.6 characterizes almost completely the spaceability of $\mathcal{U}_{A}$.

4.2. Sequence of operators from $\omega$ to $\omega$. In 2006, Bès and Conejero [8] showed that for any non-constant polynomial $P$, for any weighted shift $B_{w}$, the operator $P\left(B_{w}\right): \omega \rightarrow \omega$ possesses a hypercyclic subspace. We are interested to know which sequences of operators from $\omega$ to $\omega$ possess a hypercyclic subspace. In the following, we denote by $\left(e_{n}\right)_{n \geq 0}$ the canonical basis of $\omega$ and by $p_{n}$ the seminorms defined by $p_{n}(x)=\max \left\{\left|x_{k}\right|: 0 \leq k \leq n\right\}$.

Let $\left(T_{k}\right)$ be a sequence of continuous linear operators from $\omega$ to $\omega$. These operators can be seen as matrices $\left(a_{i, j}^{(k)}\right)_{i, j \geq 0}$, where $T_{k} e_{j}=\left(a_{i, j}^{(k)}\right)_{i \geq 0}$, i.e. the $j$ th column of $\left(a_{i, j}^{(k)}\right)$ is given by $T_{k} e_{j}$. We notice that each row of the matrix $\left(a_{i, j}^{(k)}\right)$ needs to be ultimately zero in order that $T_{k}$ is well-defined. We then denote by $c_{i}^{(k)}$ the smallest index such that for any $j \geq c_{i}^{(k)}, a_{i, j}^{(k)}=0$. In particular, it means that for any $i \geq 0$, any $x \in \omega$, the $i$ th coordinate of $T_{k} x$ only depends on the first $c_{i}^{(k)}$ coordinates of $x$.

By convention, if $A \subset \omega$, we let $A \cap \mathbb{K}^{l}:=\left\{\left(x_{k}\right)_{0 \leq k<l}:\left(x_{k}\right)_{k \geq 0} \in A\right\}$. In particular, we have

$$
T_{k}\left(\operatorname{ker} p_{N}\right) \cap \mathbb{K}^{l}=\operatorname{span}\left\{\left(a_{i, j}^{(k)}\right)_{i=0, \ldots, l-1}: j \geq N+1\right\} .
$$

We start our study of the existence of hypercyclic subspaces for $\left(T_{k}\right)$ by the following simple result:

Lemma 4.7. If $\left(T_{k}\right)$ possesses a hypercyclic subspace, then for any $N \geq 0$, any $l \geq 1$, any $K \geq 1$,

$$
\bigcup_{k \geq K} \operatorname{span}\left\{\left(a_{i, j}^{(k)}\right)_{i=0, \ldots, l-1}: j \geq N\right\} \quad \text { is dense in } \mathbb{K}^{l} .
$$

Proof. If we suppose that $\left(T_{k}\right)$ possesses a hypercyclic subspace $M_{h}$, then we know that for any $N \geq 0$, we have $M_{h} \cap \operatorname{ker} p_{N} \neq\{0\}$ and thus, for any $K \geq 1$, $\bigcup_{k \geq K} T_{k}\left(\operatorname{ker} p_{N}\right)$ is dense in $\omega$. In particular, we deduce that for any $l \geq 1$, $\bigcup_{k \geq K} T_{k}\left(\operatorname{ker} p_{N}\right) \cap \mathbb{K}^{l}$ is dense in $\mathbb{K}^{l}$. Thanks to (4.1), we therefore conclude that

$$
\bigcup_{k \geq K} \operatorname{span}\left\{\left(a_{i, j}^{(k)}\right)_{i=0, \ldots, l-1}: j \geq N+1\right\} \text { is dense. }
$$

A condition a little stronger than the condition of Lemma4.7 gives us a sufficient condition for having a hypercyclic subspace. 
Theorem 4.8. If for any $N \geq 0$, any $l \geq 1$, any $K \geq 1$, there exists $k \geq K$ such that

$$
\operatorname{span}\left\{\left(a_{i, j}^{(k)}\right)_{i=0, \ldots, l-1}: j \geq N\right\}=\mathbb{K}^{l},
$$

then $\left(T_{k}\right)$ possesses a hypercyclic subspace.

Proof. Thanks to Corollary 3.5, we can conclude if we show that there exist a sequence $\left(n_{k}\right)$ and a set $X_{0} \subset X$ such that

(1) for any $j \geq 1$, any $x \in X_{0},\left(p_{j}\left(T_{n_{k}} x\right)\right)_{k}$ is ultimately zero;

(2) for any $K \geq 1$, any $l \geq 1$, any $y_{0}, \ldots, y_{l-1} \in \mathbb{K}$, there exist $k \geq K$ and $x \in X_{0}$ such that $T_{n_{k}} x=\left(y_{0}, \ldots, y_{l-1}, *, *, \ldots\right)$.

By assumption, for any $N \geq 0$, any $l \geq 1$, any $K \geq 1$, there exists $k \geq K$ and $M \geq N$ such that

$$
\operatorname{span}\left\{\left(a_{i, j}^{(k)}\right)_{i=0 \ldots, l-1}: M>j \geq N\right\}=\mathbb{K}^{l} .
$$

We can thus construct recursively two increasing sequences $\left(n_{l}\right)$ and $\left(m_{l}\right)$ with $m_{1}=1$ such that for any $l \geq 1$,

$$
\left\{\begin{array}{l}
\operatorname{span}\left\{\left(a_{i, j}^{\left(n_{l}\right)}\right)_{i=0, \ldots, l-1}: m_{l+1}>j \geq m_{l}\right\}=\mathbb{K}^{l} \\
m_{l+1} \geq \max \left\{c_{0}^{\left(n_{l}\right)}, \ldots, c_{l-1}^{\left(n_{l}\right)}\right\} .
\end{array}\right.
$$

The second inequality implies that for any $l \geq 1$, any $x \in \omega$, the first $l$ coordinates of $T_{n_{l}} x$ only depend on the first $m_{l+1}$ coordinates of $x$ and the first equality therefore implies that for any $l \geq 1$, any $y_{0}, \ldots, y_{l-1} \in \mathbb{K}$, any $x_{0}, \ldots, x_{m_{l}-1} \in \mathbb{K}$, there exists $x_{m_{l}}, \ldots, x_{m_{l+1}-1} \in \mathbb{K}$ such that

$$
T_{n_{l}}\left(\left(x_{0}, \ldots, x_{m_{l}-1}, x_{m_{l}}, \ldots, x_{m_{l+1}-1}, *, *, \ldots\right)\right)=\left(y_{0}, \ldots, y_{l-1}, *, *, \ldots\right) .
$$

In particular, for any $l \geq 1$, we can construct a sequence $x$ block by block thanks to (4.2) such that $T_{n_{l}} x=\left(y_{0}, \ldots, y_{l-1}, *, *, \ldots\right)$ and for any $j>l, p_{j}\left(T_{n_{j}} x\right)=0$. The assumptions of Corollary 3.5 are thus satisfied for the sequence $\left(n_{l}\right)$.

Remark 4.9. The condition

$$
\operatorname{span}\left\{\left(a_{i, j}^{(k)}\right)_{i=0 \ldots, l-1}: j \geq N\right\}=\mathbb{K}^{l}
$$

is equivalent to the existence of a number $M \geq N$ such that the column rank of the matrix $\left(a_{i, j}^{(k)}\right)_{0 \leq i<l ; N \leq j \leq M}$ is equal to $l$.

Remark 4.10. In general, the converse of Theorem 4.8 is false. If we consider a dense sequence $\left(y_{l}\right)_{l>0}$ in $\omega$ and two applications $\varphi_{1}, \varphi_{2}: \mathbb{N} \rightarrow \mathbb{Z}_{+}$such that

$$
\text { for any } n, l \geq 0, \quad \#\left\{k \in \mathbb{N}:\left(\varphi_{1}(k), \varphi_{2}(k)\right)=(n, l)\right\}=\infty,
$$

then the sequence $\left(T_{k}\right)_{k \geq 1}$, defined by $T_{k}\left(e_{n}\right)=y_{\varphi_{2}(k)} \delta_{n, \varphi_{1}(k)}$, possesses $\omega$ as hypercyclic subspace but the assumption of Theorem 4.8 is not satisfied. However it seems more difficult to exhibit a counterexample in the case of an operator $T$ and its iterates. Therefore one may wonder if, in this case, the assumption of Theorem 4.8 is equivalent to having a hypercyclic subspace.

An immediate corollary of Theorem 4.8 can be stated as follows: 
Corollary 4.11. If there exist two increasing sequences of integers $\left(i_{k}\right)$ and $\left(n_{k}\right)$ such that

(i) for any $i \geq 0, c_{i}^{\left(n_{k}\right)} \rightarrow \infty$ as $k \rightarrow \infty$,

(ii) for any $k \geq 1$, any $i, j \leq i_{k}, i \neq j \Rightarrow c_{i}^{\left(n_{k}\right)} \neq c_{j}^{\left(n_{k}\right)}$, then $\left(T_{k}\right)$ possesses a hypercyclic subspace.

Proof. Let $j_{0}^{k, l}=\min _{0 \leq i<l} c_{i}^{\left(n_{k}\right)}-1$ and $j_{1}^{k, l}=\max _{0 \leq i<l} c_{i}^{\left(n_{k}\right)}$. We deduce from (ii) that for any $k \geq 1$, any $l \leq i_{k}$, the column rank of the matrix $\left(a_{i, j}^{\left(n_{k}\right)}\right)_{0 \leq i<l ; j_{0}^{k, l} \leq j \leq j_{1}^{k, l}}$ is equal to $l$. By (i), we also have that for any $l \geq 1, j_{0}^{k, l} \rightarrow \infty$ as $k \rightarrow \infty$. We conclude thanks to Theorem 4.8 and Remark 4.9 .

We remark that the conditions of Corollary 4.11 do not depend on the values of the matrices $\left(a_{i, j}^{(k)}\right)$ but only on the positions of non-zero elements of these matrices. In the case of an operator $T$ and its iterates, if we denote by $c_{i}$ the smallest index such that for any $j \geq c_{i}, a_{i, j}=0$, where $T=\left(a_{i, j}\right)_{i, j \geq 0}$, then we can obtain a necessary condition only depending on values $c_{i}$.

Corollary 4.12. Let $T: \omega \rightarrow \omega$ be a continuous linear operator. If

(i) for any $i \geq 0, c_{i}>i+1$,

(ii) for any $i \neq j, c_{i} \neq c_{j}$,

then $T$ possesses a hypercyclic subspace.

Proof. Let $n \geq 1$. By definition of $c_{i}$, we have

$$
T\left(\operatorname{ker} p_{n}\right) \subset\left\{x \in \omega: x_{i}=0 \text { if } c_{i} \leq n+1\right\}
$$

and by (ii), we also have

$$
T\left(\operatorname{ker} p_{n}\right) \supset\left\{x \in \omega: x_{i}=0 \text { if } c_{i} \leq n+1\right\} .
$$

Therefore, by (i), we get

$$
\begin{aligned}
T\left(\operatorname{ker} p_{n}\right) & =\left\{x \in \omega: x_{i}=0 \text { if } c_{i} \leq n+1\right\} \\
& \supset\left\{x \in \omega: x_{i}=0 \text { if } i<n\right\}=\operatorname{ker} p_{n-1} .
\end{aligned}
$$

We deduce that $T^{n}\left(\operatorname{ker} p_{n}\right) \supset$ ker $p_{0}$ and since

$$
T\left(\operatorname{ker} p_{0}\right)=\left\{x \in \omega: x_{i}=0 \text { if } c_{i} \leq 1\right\}=\omega,
$$

we conclude thanks to (4.1) and Theorem 4.8.

Corollary 4.12 gives us a large class of operators with hypercyclic subspaces on $\omega$. In particular, we remark that the operators $P\left(B_{w}\right)+\sum_{k=1}^{\infty} \alpha_{k} S_{w}^{k}$, where $P$ is a non-constant polynomial and $S_{w}$ is a weighted forward shift, possess a hypercyclic subspace. This contains, in particular, the result of Bès and Conejero [8].

We end this section by looking at the notion of frequently hypercyclic subspaces for operators from $\omega$ to $\omega$. Let $\left(T_{k}\right)$ be a sequence of linear continuous operators from $X$ to $Y$, where $X$ is an infinite-dimensional Fréchet space and $Y$ is a separable topological vector space. The sequence $\left(T_{k}\right)$ is said to be frequently hypercyclic if there exists a vector $x$ in $X$ (also called frequently hypercyclic) such that for any non-empty open set $U \subset X$, the set $\left\{k \geq 1: T_{k} x \in U\right\}$ is of positive lower density. We therefore say that $\left(T_{k}\right)$ possesses a frequently hypercyclic subspace if there exists a closed infinite-dimensional subspace in which every non-zero vector is frequently 
hypercyclic. The notion of frequently hypercyclic operator has been introduced by Bayart and Grivaux [2], and the notion of frequently hypercyclic subspace has been studied for the first time by Bonilla and Grosse-Erdmann [12].

Theorem 4.13. Let $f_{k, l}=\max \left\{c_{i}^{(k)}: i=0, \ldots, l-1\right\}$. If there exist $\left(d_{k}\right)_{k \geq 1}$ and $\left(N_{l}\right)_{l \geq 1}$ such that for any $k, l \geq 1$, we have

$$
\operatorname{span}\left\{\left(a_{i, j}^{(k)}\right)_{i=0 \ldots, l-1}: d_{k} \leq j<f_{k, l}\right\}=\mathbb{K}^{l}
$$

and for any $k^{\prime} \geq k+N_{l}$, we have $f_{k, l} \leq d_{k^{\prime}}$, then $\left(T_{k}\right)$ possesses a frequently hypercyclic subspace.

Proof. Let $\left(y_{l}\right)_{l \geq 1}$ be a dense sequence in $\omega$. Let $\left(f_{k, l}\right)_{k, l \geq 1},\left(d_{k}\right)_{k \geq 1}$, and $\left(N_{l}\right)_{l \geq 1}$ be sequences as given in the statement. We know (see [3], 111) that there exists a family of disjoint sets $A(l, \nu) \subset \mathbb{N}$ of positive lower density such that for any $n \in A(l, \nu)$, for any $m \in A(\lambda, \mu)$, we have $n \geq \nu$ and $|n-m| \geq \nu+\mu$ if $n \neq m$. We let $E_{l, j}=A\left(l, j+N_{l}\right)$. The goal of this proof is to construct a sequence $\left(u_{j}\right)_{j \geq 1} \subset \omega$ such that $v\left(u_{j}\right) \rightarrow \infty$ and for any $\left(\alpha_{j}\right)_{j} \in \mathbb{K}^{\mathbb{N}}$, the sequence $\sum_{j=1}^{\infty} \alpha_{j} u_{j}$ is frequently hypercyclic. To this end, we will construct the sequence $\left(u_{j}\right)_{j}$ such that for any $l, j \geq 1$, for any $n \in E_{l, j}$, for any $j^{\prime} \neq j$, we have

$$
p_{l-1}\left(T_{n} u_{j}-y_{l}\right)=0 \text { and } p_{l-1}\left(T_{n} u_{j^{\prime}}\right)=0 .
$$

We will construct these sequences block by block by going through each element in $E:=\bigcup_{l, j \geq 1} E_{l, j}$. We denote by $n_{k}$ the $k$ th element in $E$ and

$$
J_{k}=\left\{j \geq 1:\left\{n_{1}, \ldots, n_{k-1}\right\} \cap \bigcup_{l \geq 1} E_{l, j} \neq \emptyset\right\} .
$$

We remark that for any $k \geq 1$, if $n_{k} \in E_{l, j}$ and $n_{k+1} \in E_{l^{\prime}, j^{\prime}}$, then $n_{k+1}-n_{k} \geq$ $j+N_{l}+j^{\prime}+N_{l^{\prime}} \geq N_{l}$ and thus by hypothesis, we have $f_{n_{k}, l} \leq d_{n_{k+1}}$. Our construction will therefore consist at the $k$ th step in adding to some sequences $u_{i}$ a new block $u_{i, d_{n_{k}}}, \ldots, u_{i, f_{n_{k}, l}-1}$ if $n_{k} \in E_{l, j}$. In this way, if $n_{k} \in E_{l, j}$, the first $l$ coordinates of $T_{n_{k}} u_{i}$ will not be modified after the $k$ th step of our construction by definition of $f_{n_{k}, l}$. The sets $J_{k}$ will permit us to know for which sequence $u_{j}$ some blocks have already been constructed after the $(k-1)$ st step. Our construction is the following:

- If $n_{k} \in E_{l, j}$ and $j \notin J_{k}$, we let $u_{j}=\left(0, \ldots, 0, u_{j, d_{n_{k}}}, \ldots, u_{j, f_{n_{k}, l}-1}, *, *, \ldots\right)$ such that $p_{l-1}\left(T_{n_{k}} u_{j}-y_{l}\right)=0$ and for each $i \in J_{k}$, we complete $u_{i}$ by some block $u_{i, d_{n_{k}}}, \ldots u_{i, f_{n_{k}, l}-1}$ such that $p_{l-1}\left(T_{n_{k}} u_{i}\right)=0$. Such blocks exist by (4.3).

- If $n_{k} \in E_{l, j}$ and $j \in J_{k}$, we complete $u_{j}$ by some block $u_{j, d_{n_{k}}}, \ldots u_{j, f_{n_{k}, l}-1}$ such that $p_{l-1}\left(T_{n_{k}} u_{j}-y_{l}\right)=0$ and for each $i \in J_{k}, i \neq j$, we complete $u_{i}$ by some block $u_{i, d_{n_{k}}}, \ldots u_{i, f_{n_{k}, l}-1}$ such that $p_{l-1}\left(T_{n_{k}} u_{i}\right)=0$.

The condition (4.3) implies that $f_{k, l} \geq l$. Hence, $d_{k} \rightarrow \infty$ as $k \rightarrow \infty$ and thus $v\left(u_{j}\right) \rightarrow \infty$ as $j \rightarrow \infty$. We deduce that there exists a subsequence of $\left(u_{j}\right)$ and a subsequence of $\left(p_{n}\right)$ such that the condition of Lemma 3.1 is satisfied. The subspace obtained is therefore a frequently hypercyclic subspace for $\left(T_{k}\right)$.

In the case of an operator $T$ and its iterates, we obtain the following simple criterion. 
Corollary 4.14. Let $T: \omega \rightarrow \omega$ be an operator and $r \geq 1$ an integer. If $c_{0} \geq 2$ and for any $i \geq 0, c_{i+1}=c_{i}+r$, then $T$ possesses a frequently hypercyclic subspace.

Proof. By induction, we can show that $c_{i}^{(k)}=\sum_{n=0}^{k-1} r^{n}\left(c_{0}-1\right)+r^{k} i+1$ because for any $k \geq 2$, we have

$$
c_{i}^{(k)}=c_{c_{i}-1}^{(k-1)} .
$$

We thus have

$$
f_{k, l}:=\max \left\{c_{i}^{(k)}: i=0, \ldots, l-1\right\}=\sum_{n=0}^{k-1} r^{n}\left(c_{0}-1\right)+r^{k}(l-1)+1 .
$$

Therefore, letting $d_{k}=c_{0}^{(k)}-1$, we get for any $l \geq 1$,

$$
\operatorname{span}\left\{\left(a_{i, n}^{(k)}\right)_{i=0 \ldots, l-1}: d_{k} \leq n<f_{k, l}\right\}=\mathbb{K}^{l}
$$

and for any $l, k \geq 1$, for any $k^{\prime} \geq k+l$, we have

$$
\begin{aligned}
f_{k, l} & =\sum_{n=0}^{k-1} r^{n}\left(c_{0}-1\right)+r^{k}(l-1)+1 \\
& \leq \sum_{n=0}^{k+l-1} r^{n}\left(c_{0}-1\right)=c_{0}^{(k+l)}-1=d_{k+l} \leq d_{k^{\prime}} .
\end{aligned}
$$

We conclude by Theorem 4.13 ,

Corollary 4.15. Each operator $P\left(B_{w}\right)+\sum_{k=1}^{\infty} \alpha_{k} S_{w}^{k}$, where $P$ is a non-constant polynomial and $S_{w}$ is a weighted forward shift, possesses a frequently hypercyclic subspace on $\omega$.

4.3. Weighted shifts. Weighted shifts are classical examples of hypercyclic operators. In 25], a characterization of weighted shifts with hypercyclic subspaces on certain Köthe sequence spaces with a continuous norm is given. In this subsection, we complement this result by giving a characterization of weighted shifts with hypercyclic subspace of type 2 on certain Fréchet sequence spaces.

A Fréchet space $X$ is a Fréchet sequence space if $X$ is a subspace of $\omega$ such that the embedding of $X$ in $\omega$ is continuous. In other words, $X$ is a Fréchet sequence space if the convergence in $X$ implies the convergence coordinates by coordinates. A family of Fréchet sequence spaces is given by Köthe sequence spaces. Let $A=\left(a_{j, k}\right)_{j \geq 1, k \geq 0}$ be a matrix such that for any $j \geq 1$, any $k \geq 0$, we have $a_{j, k} \leq a_{j+1, k}$ and for any $k \geq 0$, there exists $j \geq 1$ such that $a_{j, k} \neq 0$. We define the (real or complex) Köthe sequence spaces $\lambda^{p}(A)$ with $1 \leq p<\infty$ and $c_{0}(A)$ by

$$
\begin{aligned}
\lambda^{p}(A) & :=\left\{\left(x_{k}\right)_{k \geq 0} \in \omega: p_{j}\left(\left(x_{k}\right)_{k}\right)=\left(\sum_{k=0}^{\infty}\left|x_{k} a_{j, k}\right|^{p}\right)^{\frac{1}{p}}<\infty, j \geq 1\right\}, \\
c_{0}(A) & :=\left\{\left(x_{k}\right)_{k \geq 0} \in \omega: \lim _{k \rightarrow \infty}\left|x_{k}\right| a_{j, k}=0, j \geq 1\right\} \text { with } p_{j}\left(\left(x_{k}\right)_{k}\right)=\sup _{k \geq 0}\left|x_{k}\right| a_{j, k} .
\end{aligned}
$$

These Fréchet spaces possess a continuous norm if and only if there exists $j \geq 1$ such that for any $k \geq 0, a_{j, k} \neq 0$.

We fix a Fréchet sequence space $X$ and a weighted shift $B_{w}: X \rightarrow X$ defined by $B_{w} e_{n}=w_{n} e_{n-1}$, where $e_{-1}=0,\left(e_{n}\right)_{n \geq 0}$ is the canonical basis in $\omega$ and $\left(w_{n}\right)_{n \geq 1}$ is a sequence of non-zero scalars. 
Theorem 4.16. Suppose that the set of finite sequences is a dense subset of $X$. If for any continuous seminorm $p$ on $X$, the set $\left\{k \geq 0: p\left(e_{k}\right)=0\right\}$ contains arbitrarily long intervals, then $B_{w}$ possesses a hypercyclic subspace of type 2.

Proof. Let $X_{0}$ be the set of finite sequences. We can prove that the assumptions of Theorem 3.3 are satisfied for $X_{0}$ and the whole sequence $(n)$. Indeed, for any $x \in X_{0}$, we remark that the sequence $\left(B_{w}^{n} x\right)_{n}$ is ultimately zero. Moreover, for any continuous seminorm $p$ on $X$, for any $n \geq 1$, we know that there exists $k \geq 0$ such that

$$
p\left(e_{k}\right)=\cdots=p\left(e_{k+n}\right)=0 .
$$

Let $x_{0}, \ldots, x_{n} \in \mathbb{K}$. We have thus that

$$
y:=\sum_{l=0}^{n} \frac{x_{l}}{\prod_{\nu=1}^{k} w_{l+\nu}} e_{k+l} \in \operatorname{ker} p \cap X_{0} \text { and } B_{w}^{k} y=\sum_{l=0}^{n} x_{l} e_{l} .
$$

Since the finite sequences are dense in $X$, we conclude by Theorem 3.3

If there exists an increasing sequence of seminorms $\left(p_{j}\right)_{j \geq 1}$ inducing the topology of $X$ such that for any $\left(x_{k}\right)_{k \geq 0},\left(y_{k}\right)_{k \geq 0} \in X$, we have

$$
\left(\left|x_{k}\right| \leq\left|y_{k}\right| \text { for any } k \geq 0\right) \Rightarrow p_{j}\left(\left(x_{k}\right)_{k \geq 0}\right) \leq p_{j}\left(\left(y_{k}\right)_{k \geq 0}\right),
$$

then it is interesting to notice that if the assumption of Theorem 4.16 is not satisfied, the existence of a weighted shift $B_{w}: X \rightarrow X$ implies that $X$ possesses a continuous norm.

Lemma 4.17. Let $\left(p_{j}\right)_{j \geq 1}$ be an increasing sequence of seminorms inducing the topology of $X$. Suppose that the set of finite sequences is a subset of $X$ and that for any $\left(x_{k}\right)_{k \geq 0},\left(y_{k}\right)_{k \geq 0} \in X$, we have

$$
\left(\left|x_{k}\right| \leq\left|y_{k}\right| \text { for any } k \geq 0\right) \Rightarrow p_{j}\left(\left(x_{k}\right)_{k \geq 0}\right) \leq p_{j}\left(\left(y_{k}\right)_{k \geq 0}\right) .
$$

If there exist a weighted shift $B_{w}$ from $X$ to $X$ and a continuous seminorm $p$ on $X$ such that the set $\left\{k \geq 0: p\left(e_{k}\right)=0\right\}$ does not contain arbitrarily long intervals, then $X$ possesses a continuous norm.

Proof. By hypothesis, there exist a continuous seminorm $p$ on $X$ and $N \geq 1$ such that for any $k \geq 0$, we have $p\left(e_{k+i}\right) \neq 0$ for some $0 \leq i \leq N$. Without loss of generality, we can suppose that $p\left(e_{0}\right) \neq 0$. Moreover, since $B_{w}$ is a weighted shift from $X$ to $X$ and $X$ is a Fréchet sequence space, we know that $B_{w}$ is continuous. Therefore, there exist $j \geq 1$ and a constant $C>0$ such that for any $k \geq 0$, for any $0 \leq i \leq N$, we have

$$
p\left(B_{w}^{i} e_{k}\right) \leq C p_{j}\left(e_{k}\right) .
$$

In particular, for any $k \geq 0$, if $p\left(e_{k}\right) \neq 0$, then $p_{j}\left(e_{k+i}\right) \neq 0$ for any $0 \leq i \leq N$. Thanks to properties of $N$ and the fact that $p\left(e_{0}\right) \neq 0$, we deduce that for any $k \geq 0, p_{j}\left(e_{k}\right) \neq 0$. Using (4.4), we therefore conclude that $p_{j}$ is a norm.

As hypercyclic subspaces of type 2 do not exist on Fréchet spaces with a continuous norm, we obtain the following characterization:

Theorem 4.18. Let $X$ be a Fréchet sequence space such that the set of finite sequences is a dense subset of $X$. Let $B_{w}$ be a weighted shift from $X$ to $X$. Let $\left(p_{j}\right)_{j \geq 1}$ be an increasing sequence of seminorms inducing the topology of $X$. Suppose that for any $\left(x_{k}\right)_{k \geq 0},\left(y_{k}\right)_{k \geq 0} \in X$, we have

$$
\left(\left|x_{k}\right| \leq\left|y_{k}\right| \text { for any } k \geq 0\right) \Rightarrow p_{j}\left(\left(x_{k}\right)_{k \geq 0}\right) \leq p_{j}\left(\left(y_{k}\right)_{k \geq 0}\right) \text {. }
$$


Q. MENET

Then the operator $B_{w}$ possesses a hypercyclic subspace of type 2 if and only if for any $j \geq 1$, the set $\left\{k \geq 0: p_{j}\left(e_{k}\right)=0\right\}$ contains arbitrarily long intervals.

Corollary 4.19. Let $X=\lambda^{p}(A)$ or $c_{0}(A)$ and $B_{w}$ a weighted shift from $X$ to $X$. The operator $B_{w}$ possesses a hypercyclic subspace of type 2 if and only if for any $j \geq 1$, the set $\left\{k \geq 0: a_{j, k}=0\right\}$ contains arbitrarily long intervals.

\section{ACKNOWLEDGMENT}

The author would like to thank the referee for valuable comments.

\section{REFERENCES}

[1] S. I. Ansari, Existence of hypercyclic operators on topological vector spaces, J. Funct. Anal. 148 (1997), 384-390.

[2] F. Bayart and S. Grivaux, Hypercyclicité : le rôle du spectre ponctuel unimodulaire, C. R. Acad. Sci. Paris 338, (2004), 703-708

[3] F. Bayart and S. Grivaux, Frequently hypercyclic operators, Trans. Amer. Math. Soc. 358 (2006), 5083-5117.

[4] F. Bayart, K.-G. Grosse-Erdmann, V. Nestoridis and C. Papadimitropoulos, Abstract theory of universal series and applications, Proc. Lond. Math. Soc. 96 (2008), 417-463.

[5] L. Bernal-González, On hypercyclic operators on Banach spaces, Proc Amer. Math. Soc. 127 (1999), 1003-1010.

[6] L. Bernal-González, Hypercyclic subspaces in Fréchet spaces, Proc. Amer. Math. Soc. 134 (2006), 1955-1961.

[7] L. Bernal-González and A. Montes-Rodríguez, Non-finite dimensional closed vector spaces of universal functions for composition operators, J. Approx. Theory 82 (1995), 375-391.

[8] J. Bès and J.A. Conejero, Hypercyclic subspaces in omega, J. Math. Anal. Appl. 316 (2006) 16-23.

[9] J. Bonet, F. Martínez-Giménez and A. Peris, Universal and chaotic multipliers on spaces of operators, J. Math. Anal. Appl. 297 (2004), 599-611.

[10] J. Bonet and A. Peris, Hypercyclic operators on non-normable Fréchet spaces, J. Funct. Anal. 159 (1998), 587-595.

[11] A. Bonilla and K.-G. Grosse-Erdmann, Frequently hypercyclic operators and vectors, Ergodic Theory Dynam. Systems 27 (2007), 383-404. Erratum: Ergodic Theory Dynam. Systems 29 (2009), 1993-1994. 
HYPERCYCLIC SUBSPACES ON FRÉCHET SPACES WITHOUT CONTINUOUS NORM 29

[12] A. Bonilla and K.-G. Grosse-Erdmann, Frequently hypercyclic subspaces, Monatsh. Math. 168 (2012), 305-320.

[13] E. Borel, Sur quelques points de la théorie des fonctions, Ann. Sci. École Norm. Sup. 12 (1895) 9-55.

[14] K. C. Chan, Hypercyclicity of the operator algebra for a separable Hilbert space, J. Operator Theory 42 (1999), 231-244.

[15] S. Charpentier, On the closed subspaces of universal series in Banach spaces and Fréchet spaces, Studia Math. 198 (2010), 121-145.

[16] S. Charpentier, Q. Menet and A. Mouze, Closed universal subspaces of spaces of infinitely differentiable functions, Ann. Inst. Fourier (Grenoble) 63 (2013), to appear.

[17] G. Godefroy and J. H. Shapiro, Operators with dense, invariant, cyclic vector manifolds, J. Funct. Anal. 98 (1991), 229-269.

[18] M. González, F. León-Saavedra and A. Montes-Rodríguez, Semi-Fredholm theory: hypercyclic and supercyclic subspaces, Proc. London Math. Soc. (3) 81 (2000), 169-189.

[19] S. Grivaux, Hypercyclic operators, mixing operators, and the bounded steps problem, J. Operator Theory 54 (2005), 147-168.

[20] K.-G. Grosse-Erdmann, Hypercyclic and chaotic weighted shifts, Studia Math. 139 (2000), 47-68.

[21] K.-G. Grosse-Erdmann and A. Peris, Linear Chaos, Springer, London, 2011.

[22] F. León-Saavedra and A. Montes-Rodríguez, Linear structure of hypercyclic vectors, J Funct. Anal. 148 (1997), 524-545.

[23] F. León-Saavedra and V. Müller, Hypercyclic sequences of operators, Studia Math. 175 (2006), 1-18.

[24] Q. Menet, Sous-espaces fermés de séries universelles sur un espace de Fréchet, Studia Math. 207 (2011), 181-195

[25] Q. Menet, Hypercyclic subspaces and weighted shifts, preprint.

[26] A. Montes-Rodríguez, Banach spaces of hypercyclic operators, Michigan Math. J. 43 (1996), 419-436.

[27] H. Petersson, Hypercyclic subspaces for Fréchet space operators, J. Math. Anal. Appl. 319 (2006), 764-782.

[28] H. N. Salas, Hypercyclic weighted shifts, Trans. Amer. Math. Soc. 347 (1995), 993-1004. 
[29] S. Shkarin, On the set of hypercyclic vectors for the differentiation operator, Israel J. Math. 180 (2010), 271-283.

Institut de Mathématique, Université de Mons, 20 Place du Parc, 7000 Mons, Belgique

E-mail address: Quentin.Menet@umons.ac.be 